\title{
Research Paper \\ Psychometric Properties of Electronic Mental Health Assessment Device for High School Students: Teacher and Parent Form
}

\author{
*Saeed Akbari-Zardkhaneh ${ }^{1} \odot$, Seyed Mohammad Shahvarani ${ }^{2}$, Nader Mansurkiaie ${ }^{3}$, Alimohammad Zanganeh ${ }^{4}$, \\ Mohsen Jallalat-Danesh ${ }^{5}$, Siamak Tahmasebi Garmtani ${ }^{6}$, Masoud Zareian ${ }^{7}$
}

\author{
1. PhD in Assessment and Measurement, Assistant Professor, Department of Education, Faculty of Education and Psychology, Shahid Beheshti University, Tehran, Iran \\ 2. PhD Student, Department of Psychology, Faculty of Education and Psychology, Shahid Beheshti University, Tehran, Iran. \\ 3. PhD in Communication Sciences, Department of Communication Studies, Faculty of Communication Sciences, Allameh Tabataba'i University, Tehran, Iran. \\ 4. PhD in Management, Physician, Social and Crime Prevention Directorate Judiciary, Tehran, Iran \\ 5. MSc., Social and Crime Prevention Directorate Judiciary, Tehran, Iran. \\ 6. PhD in Clinical Psychology, Department of Preschool Education, University of Rehabilitation Sciences and Social Welfare, Tehran, Iran. \\ 7. MSc. Student, Department of Computer Science, Faculty of Mathematics and Computer, Central Tehran Branch, Islamic Azad University, Tehran, Iran.
}

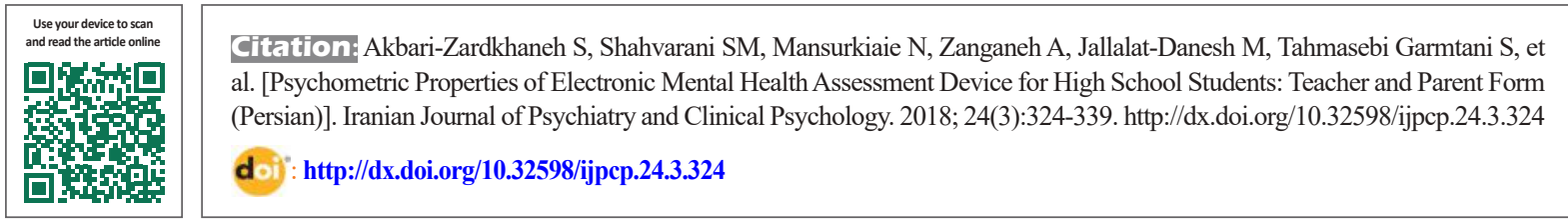

Funding: See Page 336 (c) Copyright: The Author(s)

Received: 27 Feb 2018 Accepted: 28 May 2018

Keywords: Electronic device, Factor analysis, Student's mental health, Teacher form, Parent form

\section{A B S TR A C T}

Objectives To the importance of screening students' mental health and lack of a customized tool based on prevalent psychopathological problems in students, the present study aims to develop and examine the psychometric properties of electronic mental health assessment device for high school students-parent form and teacher form.

Methods The study sample included 1697 teachers and 1186 parents of high school students of Alborz, Kermanshah, Eastern Azerbaijan, Sistan and Baluchistan, Fars, and Khorasan Razavi. Parent form and teacher form consisted of 118 and 133 items, respectively.

Results Items analysis based on 8 criteria showed that all items of teacher form and parent form had proper qualification. Explanatory factor analysis showed that the most proper structure for teacher form and parent form are 6-factor structure and 5-factor structure, respectively. Internal consistency was sufficiently good and alpha coefficients were ranged between 0.77 and 0.97 for teacher form and between 0.85 and 0.92 for parent form.

Conclusion In general, psychometric properties of both scales indicate their suitability for screening and research.

\section{Extended Abstract}

\section{Introduction}

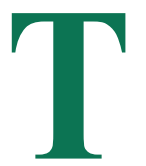

he early stage of adolescence is considered as a unique stage due to the fact that on the one hand it is adjacent to the process of transition from childhood and, on the other hand, due to maturity coincidence. Most of the well-known theories in the field of psychology of growth, directly and indirectly, have received special attention to the early adolescent age. This stage of human life, in the educational process, corresponds to the first high school (guidance school).

Changes that occur at the level of cognition, perception and interpersonal relationships of the adolescent will lead them to different issues than his childhood. As shown in

* Corresponding Author:

Saeed Akbari-Zardkhaneh, PhD

Address: Department of Education, Faculty of Education and Psychology, Shahid Beheshti University, Tehran, Iran.

Tel: +98 (21) 29905328

E-mail: akbari76ir@yahoo.com 
various studies, Several problems such as anxiety disorders, depression, hyperactivity and attention deficit disorder, impulse control, conduct disorder, lack of self-regulation, self-harm, and deficiency in the skill of doing academic assignments are prevalent in this age group. Also, the most common and complex psychosocial issues of adolescents today is child maltreatment that is associated with long-term and negative consequences in adolescence and youth. One of the main ways to prevent and reduce the complications of students' psychological problems is screening and timely referrals. Screening, by creating an opportunity for timely intervention, reduces the cost of treatment and increases the rate of recovery. There are several ways to screening the problems of children and adolescents.

One of the most effective approaches is multiple- gating screening. In this method, for more accurate screening, receives information from several sources for each person. Finally, decision is made, by summarizing the information. The importance of parents and teachers in the process of evaluation and multiple- gating screening has been shown. In one research was found that multiple scoring by parents and teachers is one of the most effective and efficient methods in the prediction of final data.

One of the essential requirements for screening programs is the availability of reliable, valid and appropriate screening tools. Various tools have been provided for this purpose in various communities. Most of the mentioned tools have merely a parent form or a teacher form, or a self-report form, and are not suitable for the multiple- gating screening system. In addition, none of them conforms to the native pattern of Iranian student societies and on the other hand, matching activities have not been performed in the country to accommodate these tools and If so, these activities are carried out in small groups and cannot be used in various communities and regions of the country. Also, the standard scores and cutoff scores that are considered for the tools are not based on the characteristics of the Iranian sample group and mostly are based on the pattern in the original version.

In addition, Today, the tendency to use electronic questionnaires has become widespread. The advantages of these tools include increasing the speed of research, facilitating the transmission of responses to data analysis software, reducing human error in data entry into software and, as a result, more precision. On the other hand, some studies believe that the quality of responses in electronic questionnaires is far better than printed questionnaires. In fact, the electronic environment causes the subject to answer more questions and commit less error. Therefore, it seems that the availability of an electronic version that is based on standardand cut-off scores according to culture and reduces human error is essential.

\section{Method}

The method of data collection in this study is descriptivesurvey. The statistical population consisted of two groups of teachers and parents of first high school students in six provinces of Alborz, Kermanshah, East Azarbaijan, Sistan and Baluchestan, Fars and Khorasan Razavi in the academic year of 2016- 2017. To prepare questions collections, a prevalent psychosocial pathology pattern was developed for students in the country. Finally, eight psychological problems including deficiency in doing academic assignments, self-harm behaviors, impulsive behaviors, and impulse control, attention deficit and hyperactivity disorder, anxiety, depression, self-regulation deficits and child abuse were selected as screening priority.

Finally, the teacher-report version reduced to 118 items and the parent-report version reduced to 133 items. In order to select a sample, six provinces that mentioned were first selected on the basis of diversity of geographical and cultural dispersion, then in each province the city with the most facilities (capital city) and the city with the least facilities in terms of socio-economic facilities were selected by targeted sampling. In the next step, three girls 'schools (elementary school, first high school and second high school) and three boys' schools (elementary school, first high school and second high school) were selected by random sampling. Finally,the opportunity of responding to research tools through computer was provided for 1186 parents and 1697 teachers from these schools in the census form.

\section{Results}

After items analysis process, parent report form reduced to 128 items. The internal consistency of the items was obtained for both versions of 0.98 . The Checking of factor analysis assumptions and Kaiser-Meyer-Olkin Measure of Sampling Adequacy Index Provided appropriate conditions for performing factor analysis of scales. In factor analysis process, the best structure that adapted with the theoretical model of mental health problems in first high school students for teacher report version was the image factoring model with Varimax rotation and reducing the components to 6 and the minimum factor load for each item of 0.3 . And for parent report version was the image factor model with Varimax rotation and reducing components to 5 and the minimum factor load for each item of 0.3 . The six-factor structure of the teacher 
report covers 60 percent and 5-factor structure parent report version covers 42 percent of the scale variance.

\section{Discussion}

The purpose of this study was to make an electronic version of the psychological health of first high school student scale in two forms of teachers and parents reports. Finally, results of items analysis, reliability coefficients of subscales, Acceptable factor validity,relatively accurate factor structure according to the theoretical model of current research, and finally the native nature of sample indicate the suitability of both versions of this electronic scale for screening and research applications in the population of first high school students in Iran.

The first high school psychological health electronic scales, with regard to its electronic content, can be used with greater ease in research and pervasive project. Therefore, the use of this scale is recommended for various research purposes such as provincial and regional screening and outbreak. Also, utilizing this tool can be very useful for more precisely preventative and therapeutic planning.

\section{Ethical Considerations}

\section{Compliance with ethical guideline}

Participants informed that extracted findings will be used for the screening of students. Also, they were free in participating in the study and completing the instruments. Therefore the complementation of instruments considers to as consonant.

\section{Funding}

The present study was conducted in line with the necessary steps to implement the national plan of social care system for students. The implementation process of this project is in the form of a coalition with participation of Shahid Beheshti University, University of Welfare and Rehabilitation Sciences, Ministry of Education, National Planning and Budget Organization, Social Assistance and Prevention of Judiciary, Interior Ministry, Imam Khomeini Executive Committee, The Ministry of Health and Medical Education, the Ministry of Labor and Social Welfare, the Welfare Organization, the Imam Khomeini Relief Committee, the Police Force, and the Counter Narcotics Headquarters.

\section{Conflict of Interest}

The authors declare no conflict of interest.

\section{Acknowledgements}

We thank all of teacher and parents for complementation of instruments. Also, we should be grateful to all schools members that have encouraged parents and teachers to participate in the study. 


\section{ويثَٔىهاي روانسنجى نسخه الكترونيكى ابزار سلامت روانشناختى دانش آموزان دوره متوسطه اول: فرم تَزارش معلم و والدين}

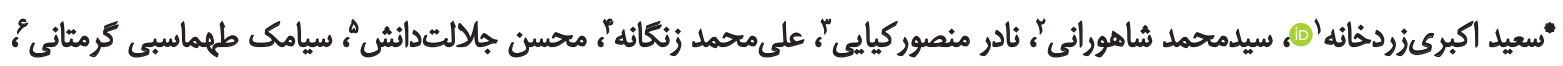
مسعود زارعيان "ميرك

ا - دكتراي سنجش و اندازهكيرى، استاديار، كروه روانشناسى تربيتى و تحولى، دانشكده علوم تربيتى و روانشناسى، دانشعاه شهيدبهشتى، تهران، ايران.

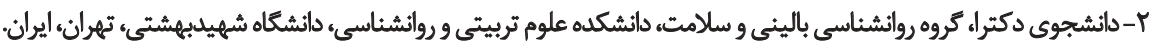

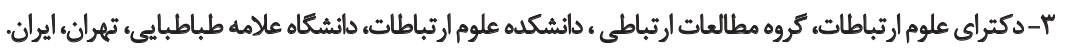

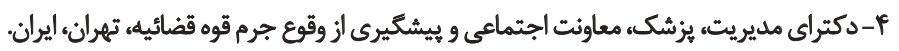

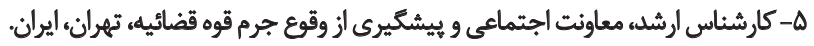

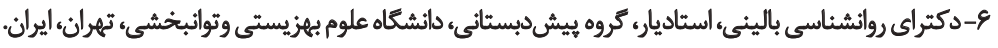

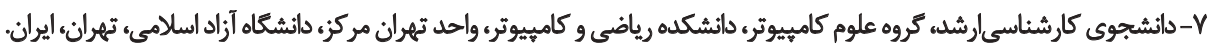

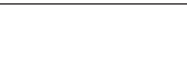

هر حله نوجواني كمتر مي شود؛ در مقابل، درك اجتماعي بيشترى

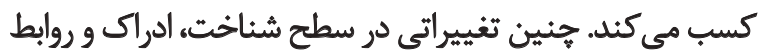

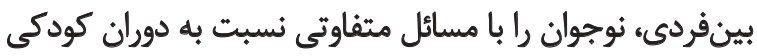

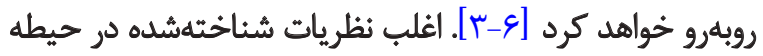
روانشناسى رشد به صورت مستقيم و غيرمستقيم توجه خاصى

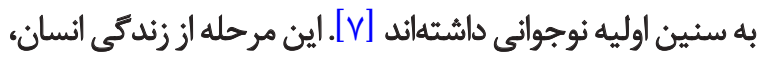

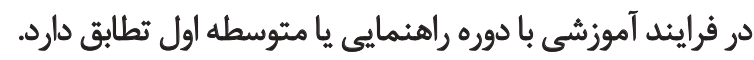

ادبيات بروهشى مربوط به شيوع اختلالات روانى در نوجوانان،
}

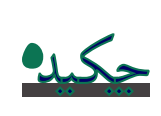

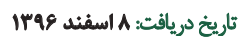

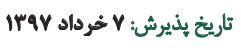

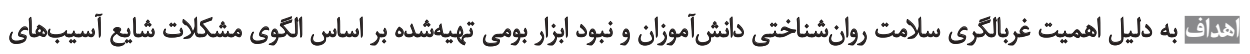

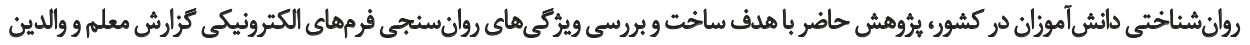

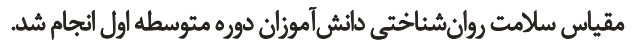

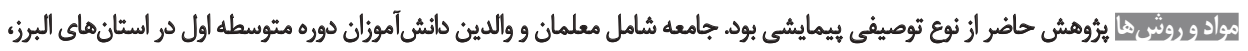

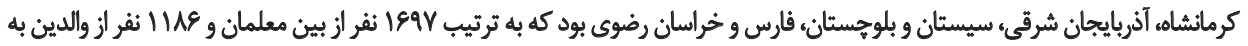

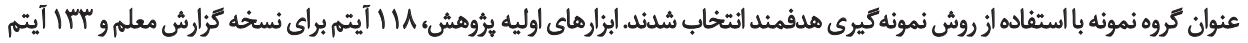

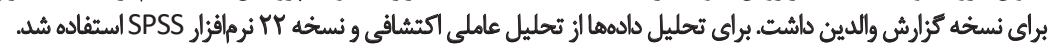

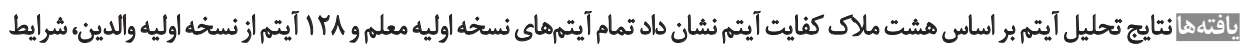

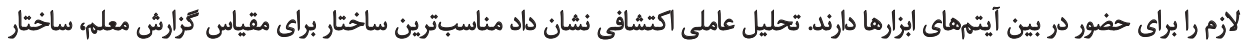

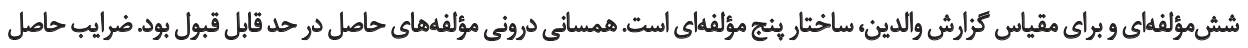

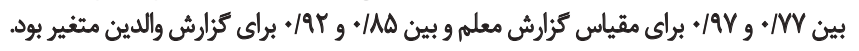

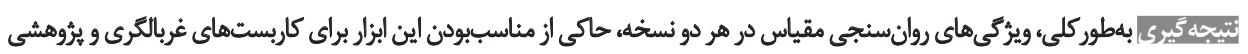

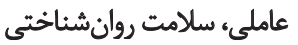
دانش آموزانه نسخه كزاري روان معلمه، نسخه كَّارش والدين

dale

مرحله اوليه نوجواني'، از طرفي به علت همرجوارى بافرايند كذار

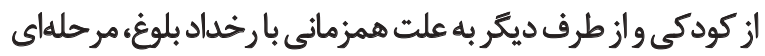

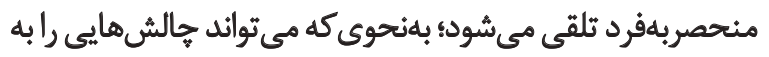

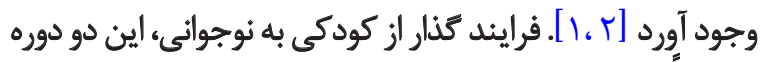

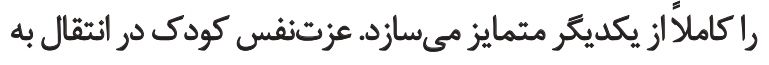

1. Early adolesence

$$
\text { ……..... }
$$

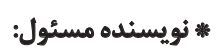

دكتر سعيد اكبرىزيردخانه

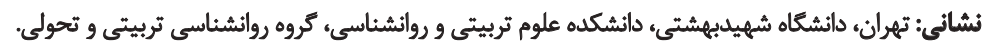

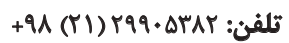
يست الكترونيكى: akbari76ir@yahoo.com 
يكى از راههاى اصلى براى بيشكيرى و كاهش عوارض مشكلات

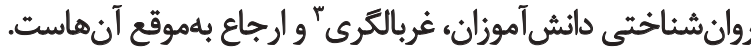

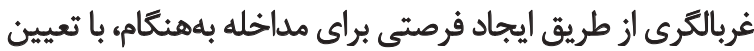

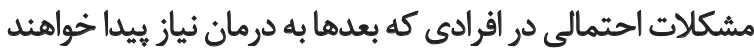

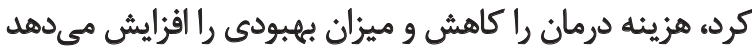

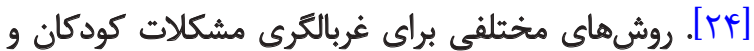

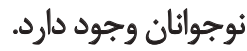

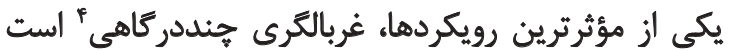

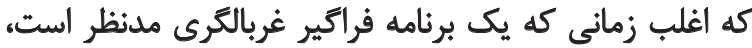

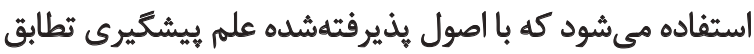

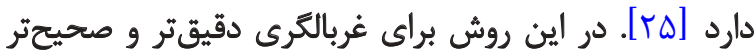

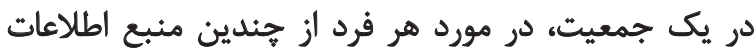

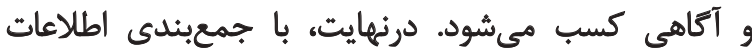

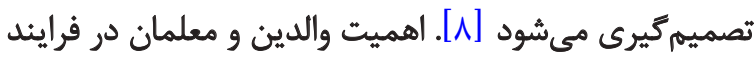

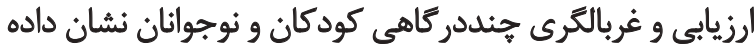

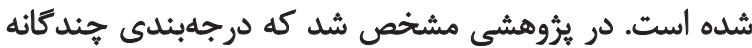

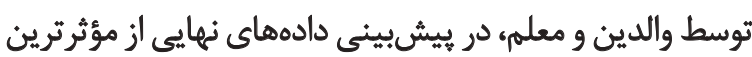
و كارآمدترين روشهاست [عبادر.

يكى از شروط اساسى برنامههاى غربالكَرى، وجود ابزار

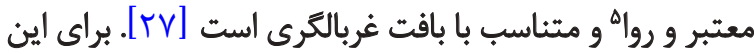

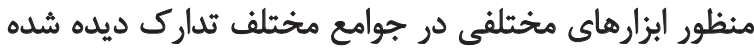

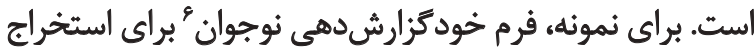

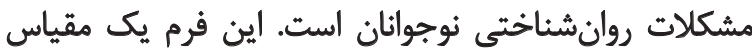

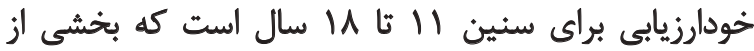

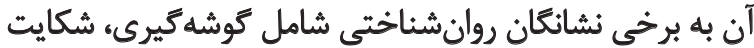

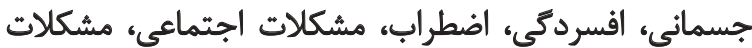

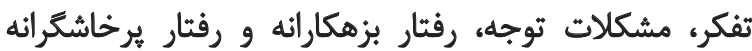

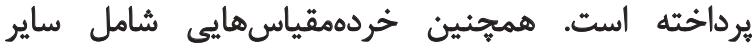
مشكلات رفتارى را نيز دربرمى ميرد.

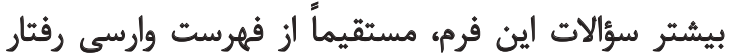

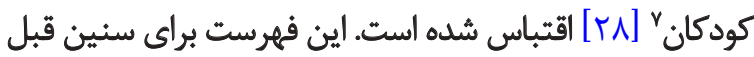

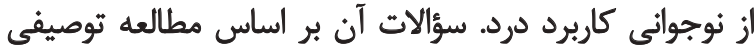

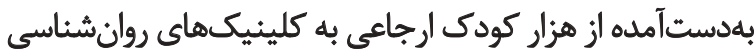

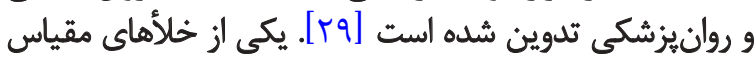

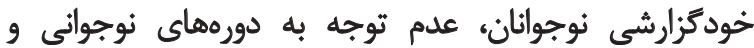

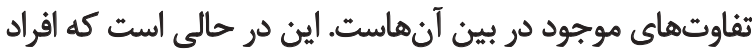

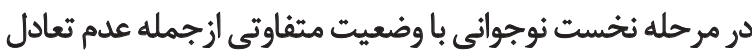

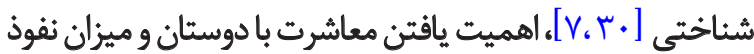

\section{Screening}

4. Multiple-gating screening

5. Reliable and valid

6. Youth Self-Report(YSR)

7. Child Behavior Checklist (CBCL)
بهور قابلملاحظهاي در سه دها كذشتثه و در سراسر جهان

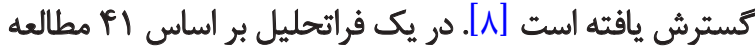

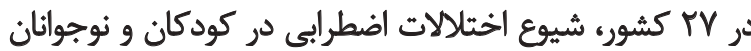

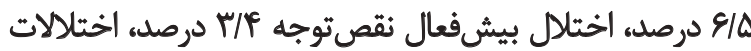

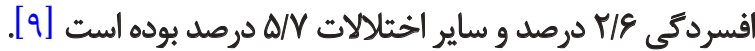

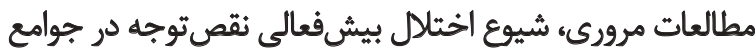

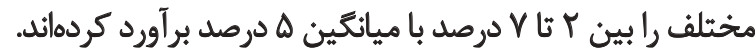

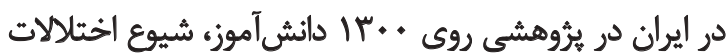

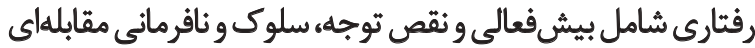

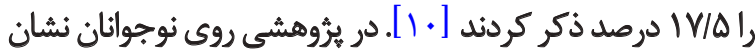

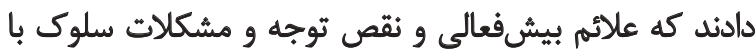

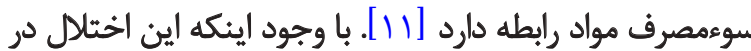

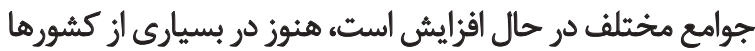

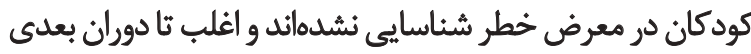

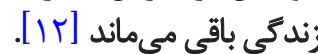

از شايعترين و بيجيدهترين مسائل روانى اجتماعى نوجوانان

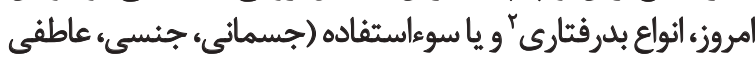

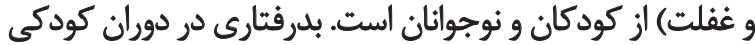

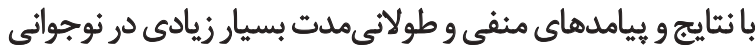

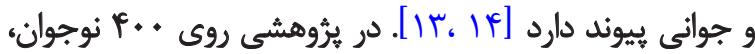

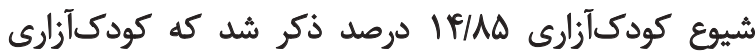

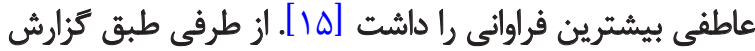

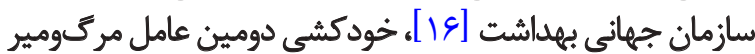

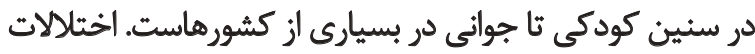

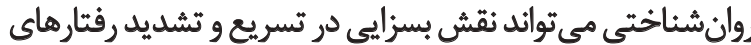

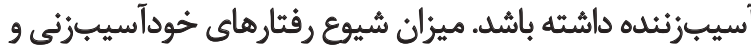

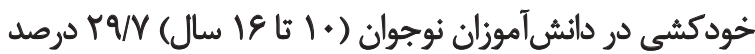

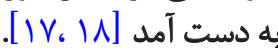

مسئله ديكرى كه ممكن است نوجوانان، بهخصوص در اوايل

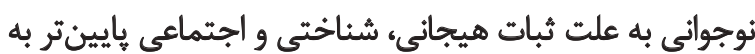

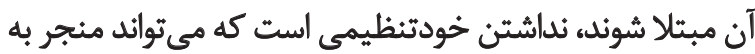

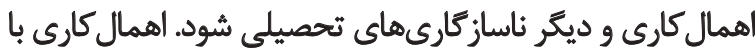

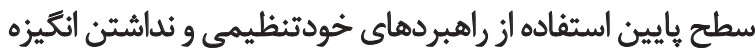

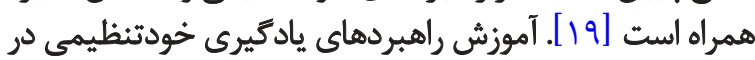

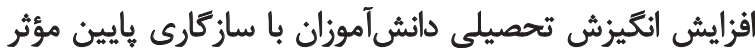

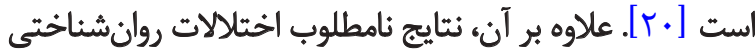

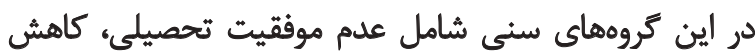

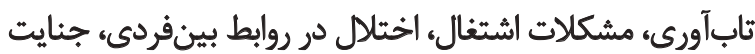

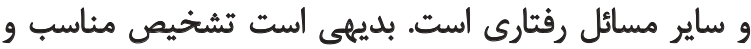

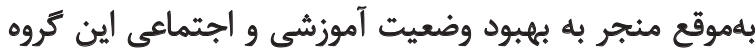

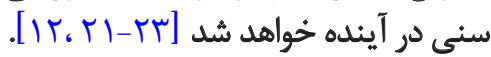




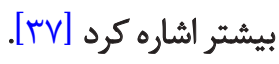

از سوى ديكر برخى يروهشها بر اين باورند كه كيفيت

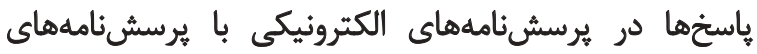

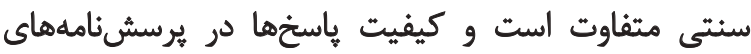

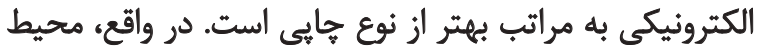

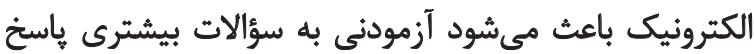

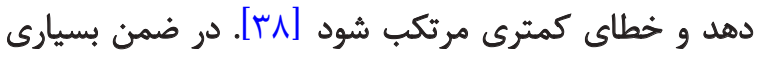

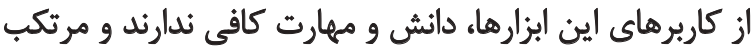

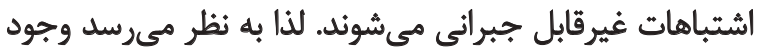

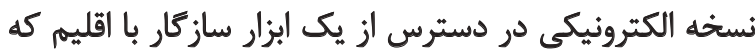

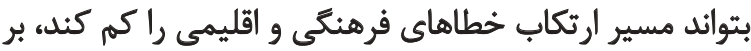

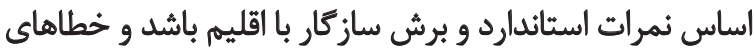

انسانى را به حداقل مقدار برساند، ضرورت و اهميت داريت دارد.

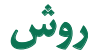

يُروهش حاضر از نوع توصيفى بيمايشى است. جامعه آمارى،

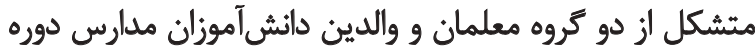

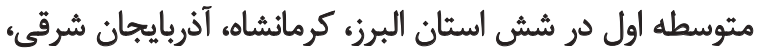

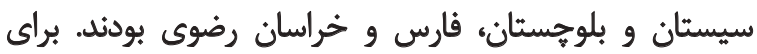

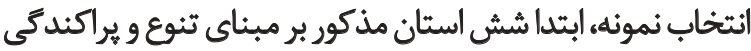

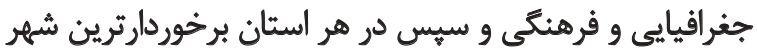

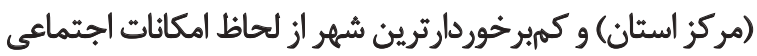
و اقتصادى به صورت نمونه

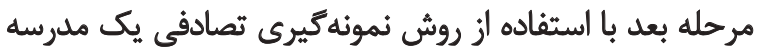

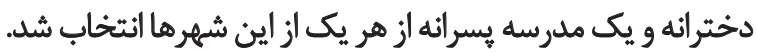

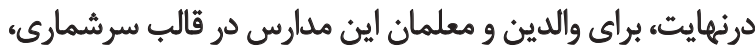

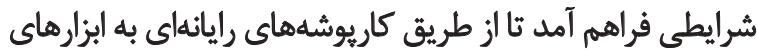

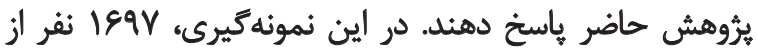

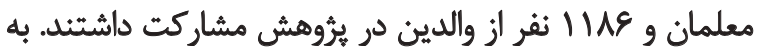

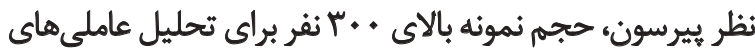

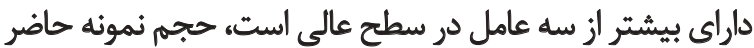

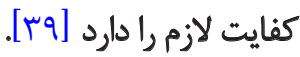

مطالعه حاضر در راستاى اقدامات زميئهاى لازم براى اجراى طرح ملى نظام مراقبتهاى اجتماعي دانشي آموزان (نماد) انجام الزام

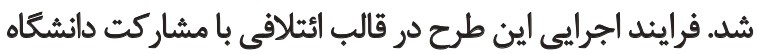

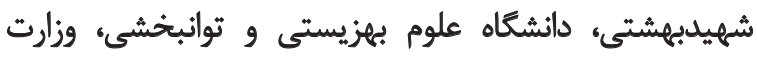

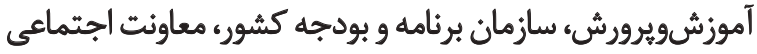

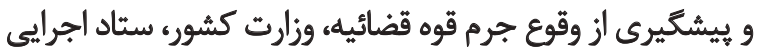

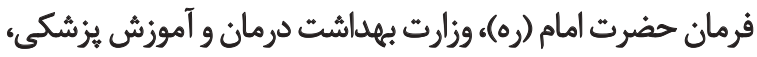

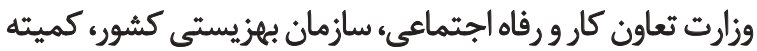

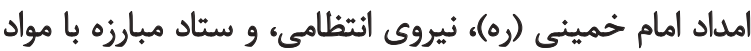

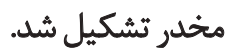

در يُؤهش حاضر، دو فرايند جداكانه براى تزارش والدين
والدين نسبت به دوره كودكى و سنين بالاتر [اسـ] روبهرو هستند

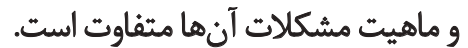

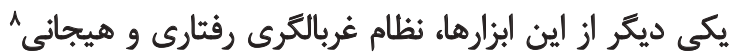

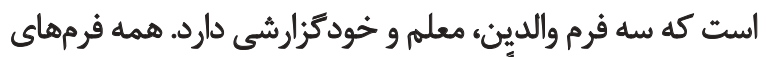

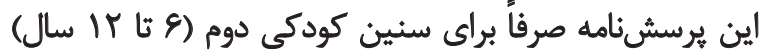

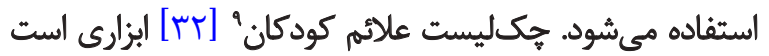

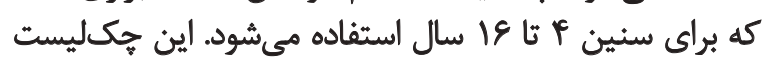

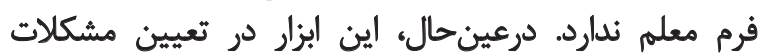

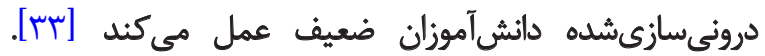

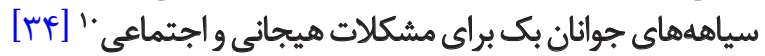

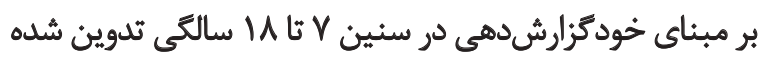

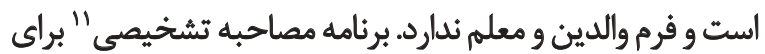

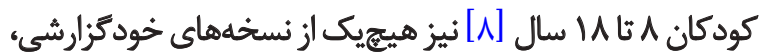

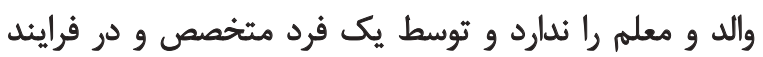
مصاحبه رودررو تكميل مي شود ودي.

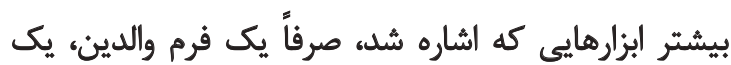

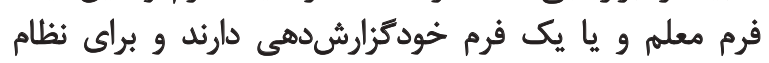

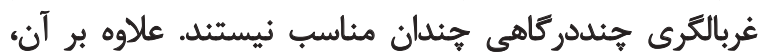

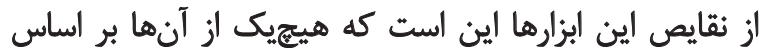

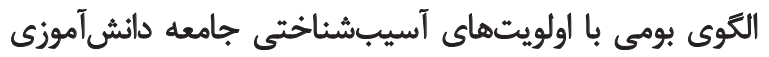

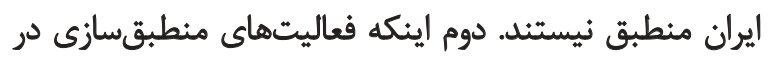

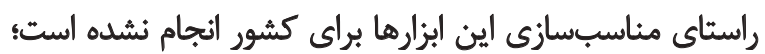

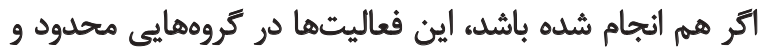

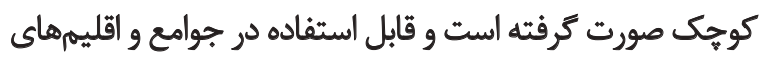

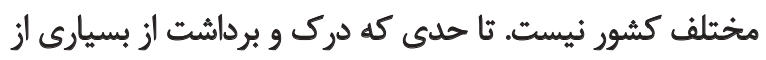

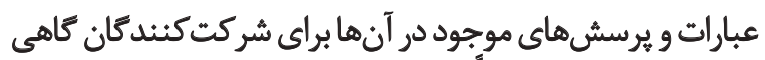

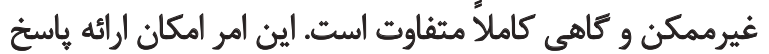

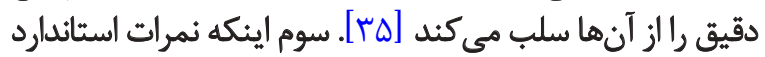

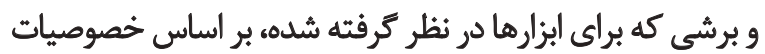

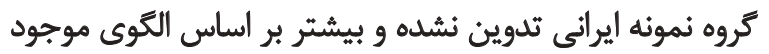
در نسخه اصلى است.

از طرف ديكر، امروزه كرايش به استفاده از برسشنامهاى

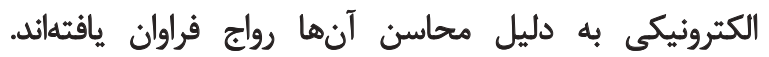

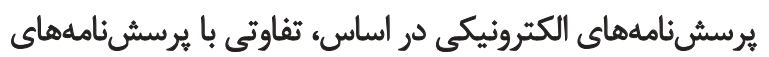

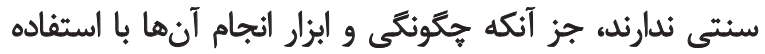

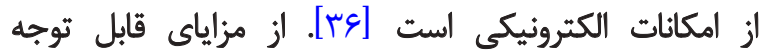

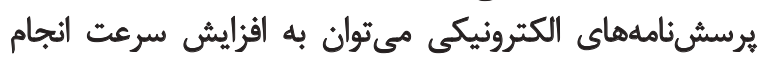

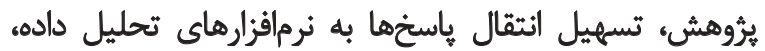

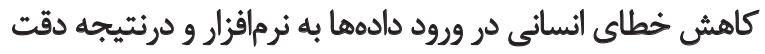

8. Behavioral and Emotional Screening System (BASC-2 BESS) 9. Pediatric Symptom Checklist (PSC)

10. Beck Youth Inventories for emotional-social problems

11. Diagnostic Interview Schedule (DIS) 
بستر اينترنت، به ابزار هاسخ داده شد. همجينين در فرايند تحليل

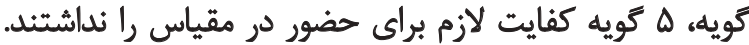

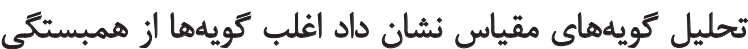

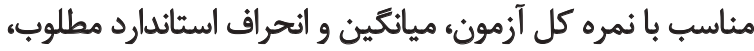

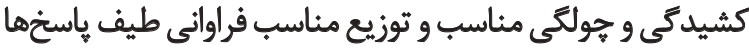

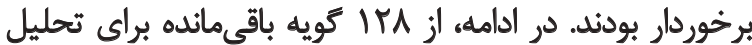
عاملى اكتشافي و بررسى ساختار درون مقياس استفاده شد.

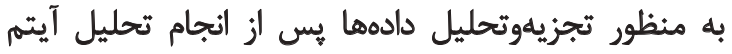

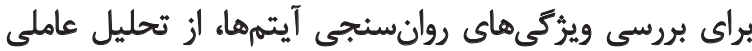

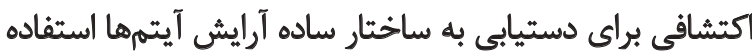

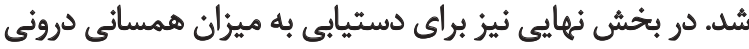

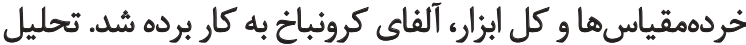

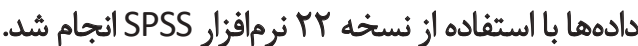

ياقتهها

$$
\text { تحليل عاملى نسخه تَّز ارش معلم }
$$

يس از فرايند تحليل گويهها، همسانى درونى نسخه الكترونيكى

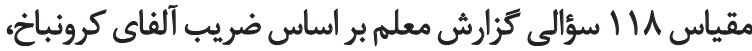

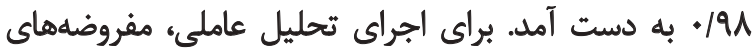

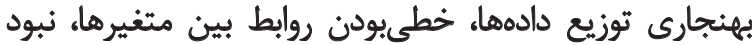

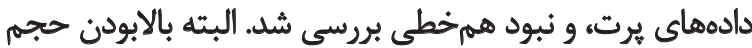

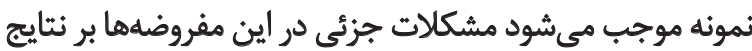

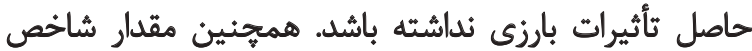

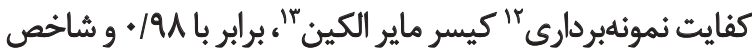

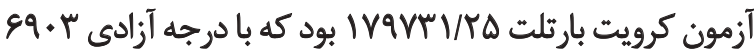

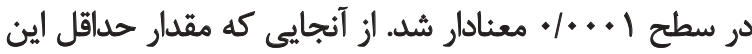

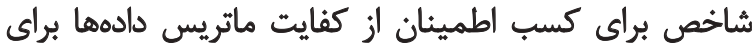

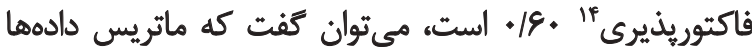
مفروضه لازم رابراي انجام تحليل عاملي دائ دراد. لذا شرايط مائ مناسب

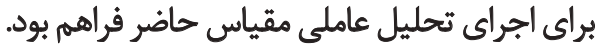
براى استخراج عوامل موجود در نسخه الكترونيكى مقياس

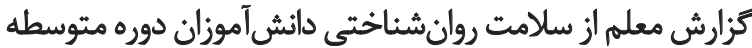

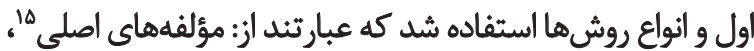

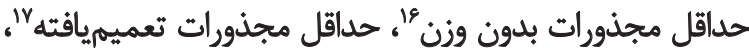

12. Sampling adequacy significance test

13. Kaiser-Meyer-Olkin measure of sampling adequacy (KMO)

14. Factorability

15. Principle components

16. Unweighted least squares

17. Generalized least squares
و كَزارش معلمان طى شله است. يیيش از تهيه بانك سؤالات

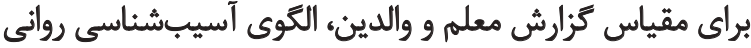

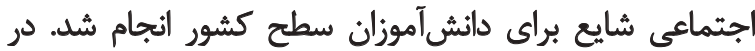

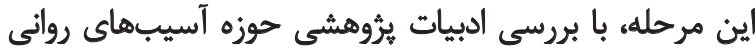

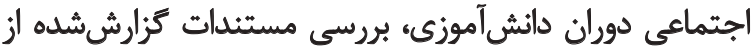

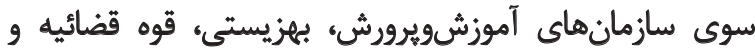

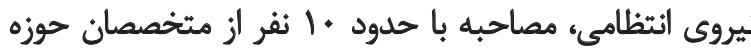

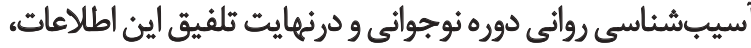
هشت آسيب روانشناختى شامل نقص دراني درانياني انجام تكاليف

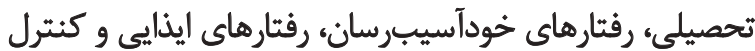

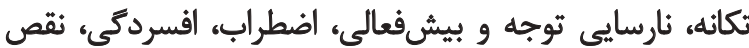

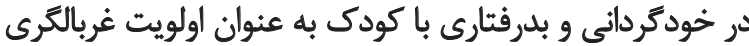

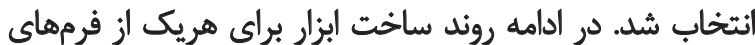

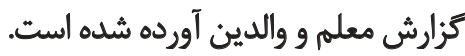

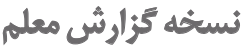

با توجه به تعيين هشت آسيب شايع در دانشآموزان، بانك

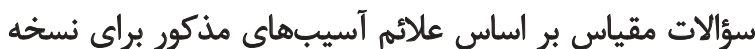

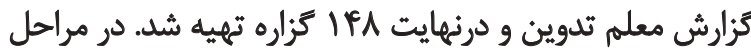

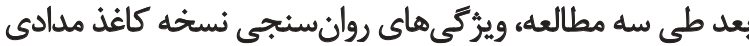

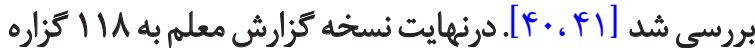

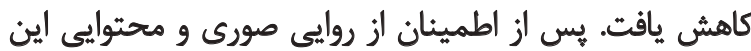
مقياس، در جامعه معلمان دوره متوسطه اول إل و در بر بستر اينترنته

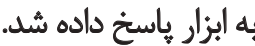

در مرحله بعده براى انجام فرايند تحليل كويه، هشت ملاك

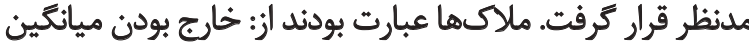

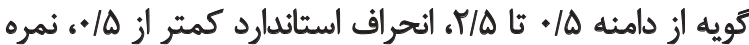

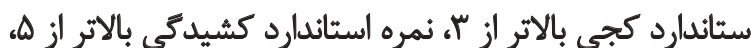

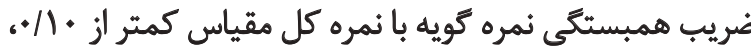

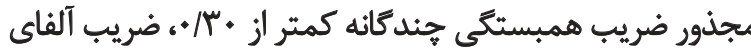

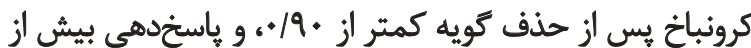

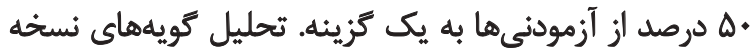
كزارش معلم نشان داد اغلب كويهها كفايت لازم رائ راد ادارند. درنتيجه

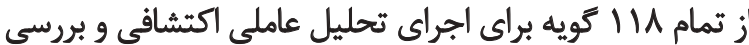
ساختار درون مقياس استفاده شداي تراي

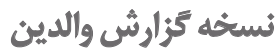

در اين نسخه بر اساس التوى آسيبشناسى اين مطالعه، ابتدا

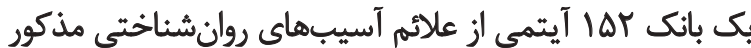

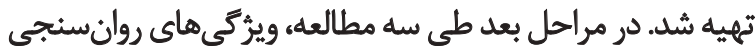

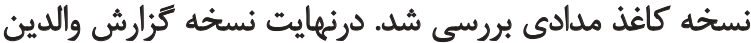

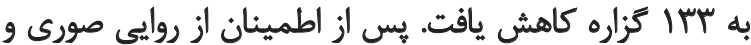

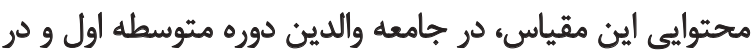


جدول ا. مؤلفهاى حاصل از تحليل عاملى نسخه الكترونيكى مقياس كزارش معلم از سلامت روانشناختى دانشآموزان در دوره متوسطه اول

\begin{tabular}{|c|c|c|c|}
\hline درصد تيين واريانس تراكمى & درصد تبيين واريانس & ارزش ويزٔه & عامل \\
\hline FI/ve & five & $r q / r \Lambda$ & 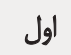 \\
\hline$\Delta .199$ & NaY & $1 . / \Delta P$ & دوم \\
\hline$\Delta F / M$ & $P / M$ & p/qr & سوم \\
\hline$\Delta V / F \Delta$ & $r / \Delta V$ & $r / . r$ & جهارم \\
\hline$\Delta Q / 11$ & $1 / M$ & $r / . \varphi$ & بينجم \\
\hline $8 . / 4$. & $1 / r r$ & $1 / \mu \varphi$ & ششم \\
\hline
\end{tabular}

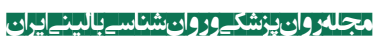

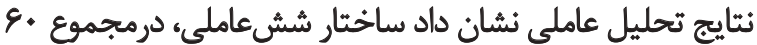

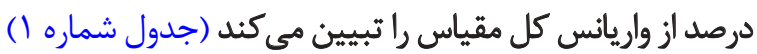

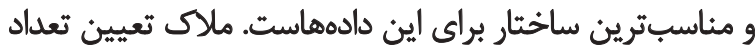

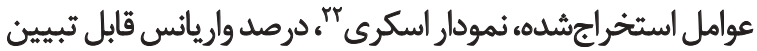
توسط مؤلفهها و محتواي كويههاي هر مؤلفه بوداري.

بررسى نتايج تحليل عاملى اكتشافى نشان داد مؤلفهانهاى

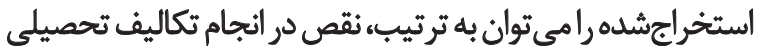

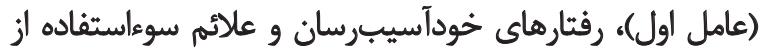

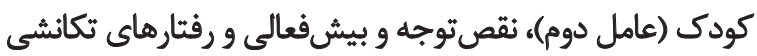

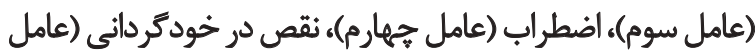

22. Scree plot

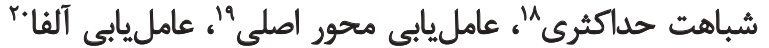

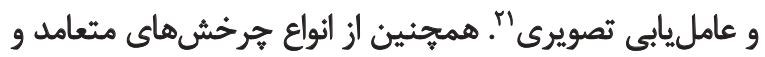

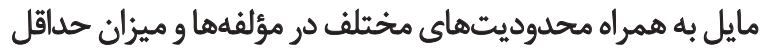
بار عاملى متغيرها استفاده شداه.

درنهايت، بهترين ساختارى كه با التوى نظرى مشكلات

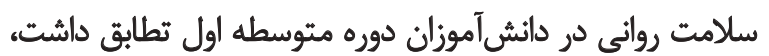

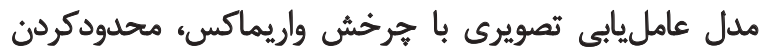

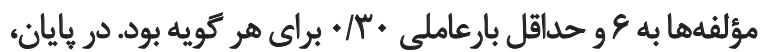

18. Maximum likelihood

19. Principle axis factoring 20. Alpha factoring

21. Image factoring

جدول r. محتوا و ويثُكى هاى روانسنجى بئج كويه از هر يك ازخردمقياسها و ضرايب همسانى درونى خردممقياسهاى نسخه الكترونيكى مقياس كزارش معلم

\begin{tabular}{|c|c|c|c|c|c|c|}
\hline ضريب & همبرهتكى با & 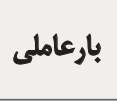 & (انحرافمعيار) & محتوا & شماره & عامل \\
\hline \multirow{5}{*}{.$/ 9 V$} & . /AT &.$/ 94$ &.$/ N 9(. / 9 Y)$ & بى بقتى در انجام تكاليف تحصيلى & r & \multirow{5}{*}{ يكم } \\
\hline & - /AP & /NE &.$/ \pi T(\cdot / A V)$ & نيمهتمام رها كردن تكاليف & ra & \\
\hline & . $/ A F$ & ./AV &.$N \varepsilon(\cdot / 9 Y)$ & نياز به كمك براى شروع تكاليف & re & \\
\hline & ./AF & . & $\cdot / 1 /(\cdot / 9))$ & اشتباه زياد در انجام تكاليف & ve & \\
\hline & $\cdot / A F$ & $\cdot / N$ &. $\mid 98(. / 94)$ & بى علاقكى به امور تحصيل & $1 . v$ & \\
\hline \multirow{5}{*}{.$/ 94$} & + &.$/ \Delta A$ &.$/ 18(+/ / 9 q)$ & رفتارهاى خطرناى أز ديد ديكران & 8 & \multirow{5}{*}{ دوم } \\
\hline & .191 &.$/ 199$ &.$/ 1 Y(\cdot / \% T)$ & خود را وادار به استفراغ كردن & ro & \\
\hline & .191 & $+N$ &.$/ N(\cdot / F A)$ & بيان آزار جسمى و جنسى & १ & \\
\hline &.$/ 9 F$ &.$/ 48$ & $\cdot / \pi(+/ \Delta F)$ & كنجكاوى در مورد موضوع جنسى & 19 & \\
\hline &. $\mid 8 \Lambda$ & . &.$/ 14(\cdot / 49)$ & سرووضع نامناسب در كلاس و مدرسه & 111 & \\
\hline
\end{tabular}




\begin{tabular}{|c|c|c|c|c|c|c|}
\hline ضريب & همبستكي با كل & 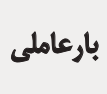 & (انحرافمعيار) & هـتوا & شماره & 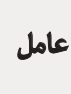 \\
\hline \multirow{5}{*}{.$/ 9 V$} & $\cdot M r$ &.$/ 4 \Delta$ &.$/ T \Delta(\cdot / 8 \Lambda)$ & حركت بدون هدف دستها و باها & r & \multirow{5}{*}{ 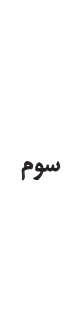 } \\
\hline & -NO &.$/ 4 \alpha$ & $\cdot / N \varphi\left(\cdot / N^{\varphi}\right)$ & ناتواني در تفريح بي سروصدا & ir & \\
\hline & $\cdot M r$ & $.18 v$ & $\cdot / \pi r(\cdot / 8 \mathrm{~N})$ & بالا و بإيين بريدن عداوم در كلاس & $r$ & \\
\hline & .180 & - lar & $\cdot / T \&(\cdot / \Delta V)$ & انثقام از ديكران & ז & \\
\hline & $\cdot M$ & .198 & $\cdot / F+(\cdot / N+)$ & زود از كوره دررفتن & $1+1$ & \\
\hline \multirow{5}{*}{$+\mathbb{N} E$} & $. / \Delta \mid$ &.$/ \% \Delta$ & $\cdot|A|(\cdot \mid M)$ & حساس و زودرنج بودن & $\pi$ & \multirow{5}{*}{ جهار } \\
\hline & $+N$ &.$/ \pi$ & $+/ F i(\cdot / N)$ & ترس از تعامل كردن & ar & \\
\hline & $\cdot M$ &.$/ 4 A$ & $+/ \Delta \mid(\cdot / V \Delta)$ & واهمه از روبلرو شلن با افراد مهم & 99 & \\
\hline & . &.$/ 4 T$ & $\cdot / r \Delta(\cdot / 89)$ & ترس از شركت در جشنها & $\mathrm{ra}$ & \\
\hline &.$/ \Delta$ &.$/ 4$ & $+/ \mu \mid(\cdot / 8+)$ & عوض كردن مداوم دوستان & A. & \\
\hline \multirow{5}{*}{$\cdot M$} &.$/ F \Delta$ & .10 & $V / \Delta(1 / 1 \cdot)$ & ثاتوانى در برناملهريزى درسى بهتئهايى & $\wedge$ & \multirow{5}{*}{ هُجْم } \\
\hline & .109 & Igr & $V / q(1 / 1 \cdot)$ & تمايل نداشتن به انجام هسثقل فعاليتها & $\Delta \Delta$ & \\
\hline &.$/ \Delta T$ & .101 & $1 / 8(1 / M)$ & نداشتن تواثاييى سازكارى با شرايط & gr & \\
\hline & $/ \Delta \mu^{\prime}$ & .188 & $1 / 9(1 /+1)$ & بيان نكردن فكر و احساس در كلاس & $\wedge$ & \\
\hline &.$/ 8 r$ &.$/ v e$ & $V / N(V / * V)$ & ثاتوائى انطبات فكر و احساس با هوقعيت & 91 & \\
\hline \multirow{5}{*}{$+/ 19$} & .189 &.$/ \mu$ & $\cdot|\Delta|(\cdot / 1 \cdot)$ & كمانرزى بودن & re & \multirow{5}{*}{ 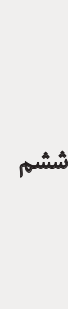 } \\
\hline & .188 &.$/ 4$ & $\cdot / r N(\cdot 189)$ & بيىيلي براى تفريحات كروهي & 94 & \\
\hline & $\cdot M$ & - pra & $\cdot /$ $(\cdot / M)$ & بى ميلى به كارهاى مورد علاقه ديكران & $1 \cdot 1$ & \\
\hline & . $M a$ &.$/ 4 q$ & $\cdot / P F(\cdot N E)$ & نكاه سرد و بي هيجان در كلاس & $1 \cdot 0$ & \\
\hline &.$/ \Delta V$ & 每 & $\left.\cdot / \pi r(\cdot / \Delta)^{2}\right)$ & احساسات منفى به خود & $111 \%$ & \\
\hline
\end{tabular}

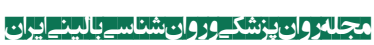

همسانى درونى كل خردهمقياس را نشان مىدهد. جدول ينجم) و افسردگى (عامل ششم) نام نهاد.

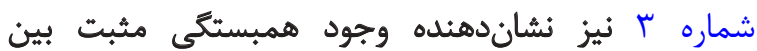
خردهمقياس هاى ابزار است.

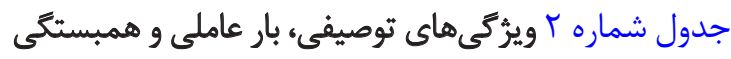

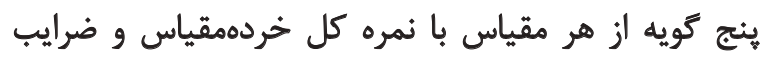

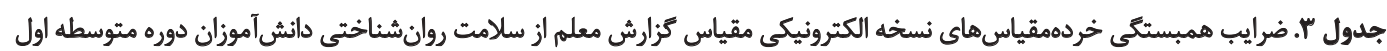

\begin{tabular}{|c|c|c|c|c|c|c|}
\hline$\bullet$ & $\varepsilon$ & $r$ & Y & 1 & مؤلفهنها & \\
\hline & & & & 1 & نقص در أنجام تكاليف تحصيلى & 1 \\
\hline & & & 1 & •/rq"*** & رفتارهاى خودأسيبرسان و عالاتم سوعرفتار با كودى & r \\
\hline & & 1 & $. / e v e=$ & $\cdot / N \cdot *$ & بيش فعالى و نقص توجه و رفتارهاي تكانشى & r \\
\hline & 1 & .198** & . $/ \Delta r^{n+*}$ & $\cdot(\mathrm{V})^{* *}$ & اضطراب & f \\
\hline 1 & $.1 \cdot \%$ & $+1.8 * *$ & $+1.8 * *$ &.$/ 1 r^{* * *}$ & نقص در خودكرداني & $\Delta$ \\
\hline.$/ 1 Q^{* * *}$ & $\cdot \mathrm{W}^{* * *}$ & •le & 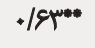 & $\cdot / r^{* * *}$ & افسرد5ى & \& \\
\hline
\end{tabular}


جدول F. مؤلفههاي حاصل از تحليل عاملى نسخه الكترونيكى مقياس تزارش والدين از سلامت روانشئاختى دانش آموزان در دوره متوسطه اول

\begin{tabular}{|c|c|c|c|}
\hline درصد تيين واريانس تراكمى & درصد تييين واريانس & ارزشّ ويُّه & عامل \\
\hline$r \cdot / 10$ & $r+/ 10$ & TNA9 & 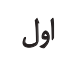 \\
\hline mefeq & r/Af & $\Delta / A 1$ & s \\
\hline wher & $T / M^{\infty}$ & $r / \Delta \cdot$ & 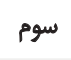 \\
\hline mq/A. & $r / M V$ & $r / \bullet r$ & جهار \\
\hline PIfs. & $1 / A$ & $r / T r$. & نينجم \\
\hline
\end{tabular}

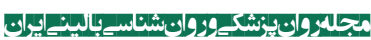

فاكتوريذيرى" "ع/ • است، مىتوان كفت كه ماتريس دادهها

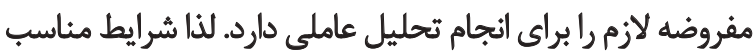

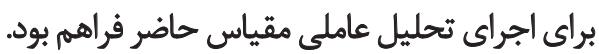

براى استخراج عوامل موجود در نسخه الكترونيكى مقياس

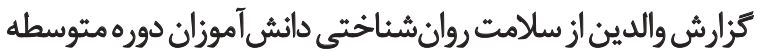

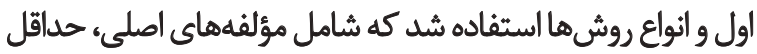
مجذورات بدون وزن، حداقل مجذورات تعميميافته، شباهت

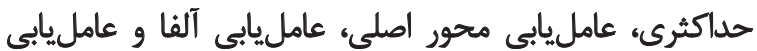

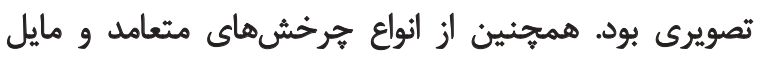

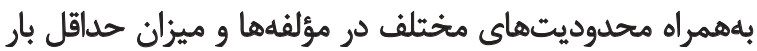

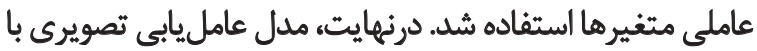

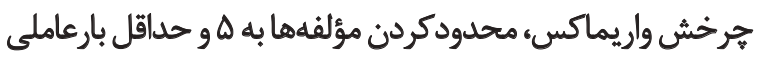

23. Factorability
تحليل عاملى نسخه كَّزارش والدين

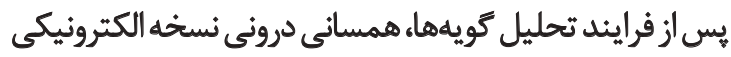

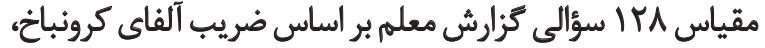

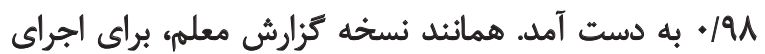

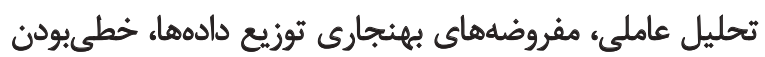

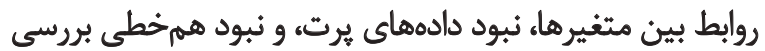

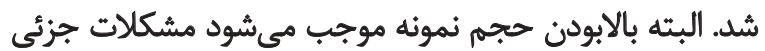

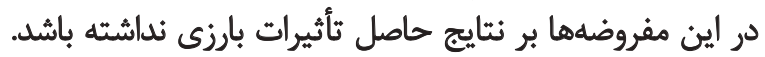

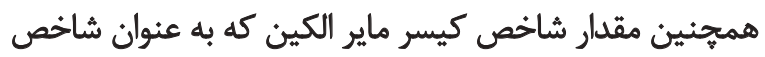

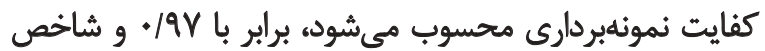

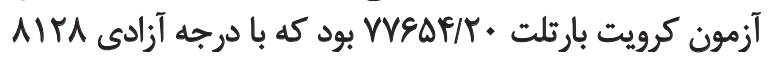

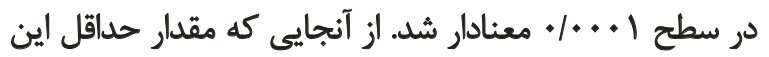

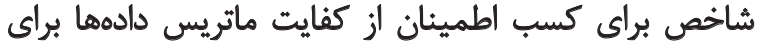

جدول ه. محتوا و ويزٔكى هاى روان سنجى كويهها به تفكيك خردهمياس ها و ضرايب همسانى درونى خردهمياس هاى نسخه الكترونيكى مقياس كزارش والدين

\begin{tabular}{|c|c|c|c|c|c|c|}
\hline ضريب ألفا & نمبره كلئي با نمل & بارعاملى & ميانكين (انحراف معيار) & 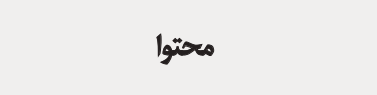 & شماره & 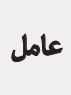 \\
\hline \multirow{5}{*}{.$/ 94$} &. $\mid+1$ & . $/ \Delta T^{\mu}$ & $\cdot / M)\left(\cdot / M^{i}\right)$ & بيان آلزار جنسى و جسمى & 9 & \multirow{5}{*}{$\overline{5}$} \\
\hline & $\cdot(\Delta)^{f}$ & $.10 \%$ & $\cdot M \pi(\cdot|8|)$ & رقثتارهاي جنسى ثاهتناسب باسن & r & \\
\hline &. $\operatorname{lag}$ & $.0 / 8 r$ &.$/ M P(\cdot / 8 T)$ & واردكردن اشياى نوكتيز در بلن خود & $N$ & \\
\hline &. $\operatorname{lat}$ & . $/ \Delta r$ & $\cdot / r \cdot(\cdot / \& 8)$ & تهلديد به آسيب رسائلن عمدى به خود & $1 \cdot r$ & \\
\hline & \multirow[t]{2}{*}{$.1 \Delta 8$} &.$/ \Delta$ & $\cdot M T(\cdot / \Delta \Lambda)$ & سرووضع نامناسب & 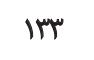 & \\
\hline \multirow{5}{*}{.194} & &.$/ \Delta \Delta$ & $\cdot 199(\cdot / M)$ & بيى دقتى در انجام تكاليف تحصيلى & $\checkmark$ & \multirow{5}{*}{2} \\
\hline & .109 & r & $+19 V(+/ A V)$ & بىعلاقكى به انجام تكاليف كلاس & 部 & \\
\hline & $.19 \mathrm{~V}$ & $\cdot / \Delta A$ & $\cdot \mid 8 \Delta(\cdot / M)$ & نياز به يادآورى براى انجام تكاليف & va & \\
\hline & 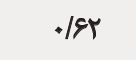 &.$/ \Delta \Delta$ & $\cdot / V \Delta(+/ \wedge 9)$ & سرعت بايين در انجام تكاليف & $1+1$ & \\
\hline & $.18 \Delta$ &.$|8|$ & $\cdot \operatorname{Aor}(\cdot / A))$ & بي علاقكى به امور تحصيل & irr & \\
\hline
\end{tabular}




\begin{tabular}{|c|c|c|c|c|c|c|}
\hline ضريب آلفا & تمبره كلكي با & بارعاملى & ميانئين (انحراف ميار) & محتوا & شماره & 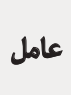 \\
\hline \multirow{5}{*}{. /AV } &.$/ P V$ & . Ra & $\cdot / F \&(\cdot / V \Lambda)$ & حركت دستوياها بلون هدف & r & \multirow{5}{*}{$\frac{2}{2}$} \\
\hline & $\cdot / \Delta V$ & . $/ \Delta T$ & $\cdot / A \cdot(\cdot / 9 V)$ & حرف زدن زياد & $P Q$ & \\
\hline & . lar & . Mt &.$/ W(\cdot / 99)$ & حركت بلطور مرتب & QI" & \\
\hline & . $/ a r$ &.$/ 4$ & $\cdot / \Delta V(\cdot / 19)$ & بالا و إيين يريلن در خانه & Ar & \\
\hline & .18 & $4 / 49$ & $\cdot|8|(\cdot / A r)$ & سرييجيى از دستورات واللدين & 11. & \\
\hline \multirow{5}{*}{.$/ 14 \Delta$} &.$/ 41$ & . &.$/ 91(1)$ & ناراحتى شديد در زمان جدايعى & f & \multirow{5}{*}{ 售 } \\
\hline & . $/ 0$ r & 吾 & $I / K g(V / \backslash \Delta)$ & ترس أز آسيبديلن والدين & r & \\
\hline &.$/ 4 A$ &.$/ \Delta$ & $\cdot / M(\cdot / M)$ & دشوارى در تنهها هاثدن & eV & \\
\hline & .10 &.$/ P A$ & $\cdot / M(Y / \cdot Y)$ & نكراتي در مواقع جدايى از مادر & $w$ & \\
\hline &.$/ p q$ &.$/ R A$ & $\cdot / R V(\cdot / N A)$ & ترس از حضور در مكانهاي عموهي & 91 & \\
\hline \multirow{5}{*}{.$/ M$} &.$/ p q$ &.$/ M$ &.$/ N E(\cdot / 9 Y)$ & رفتارهاي يرخطر & $\Delta$ & \multirow{5}{*}{ 承 } \\
\hline &.$/ \Delta Y$ &.$/ 2 V$ & $\cdot M(\cdot / a \cdot)$ & انرزى كم & rq & \\
\hline & $.1 \Delta 5$ & 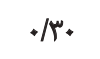 & $\cdot / \pi+\%(\cdot / 9 \cdot)$ & يرخورى يا بيىاشتهايع شديد & $v^{f}$ & \\
\hline & . &.$/ 4 T$ & $\cdot / E \cdot(\cdot / M)$ & بيان بىمحبتى خانواده يا ملربسه & $i r$ & \\
\hline & .180 &.$/ 4 q$ & $\cdot / \Delta T^{H}(\cdot / A r)$ & احساسات منفى به خود & IrA & \\
\hline
\end{tabular}

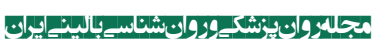

نقص در انجام تكاليف تحصيلى (عامل دوم)، بيشفعالى و نقص

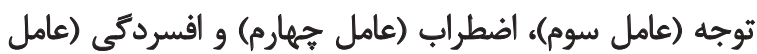

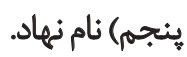

جدول شماره له ويزٔكى هاى توصيفى، بارعاملى، همبستَّى ينج

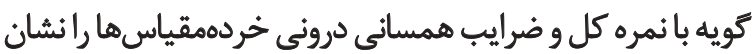

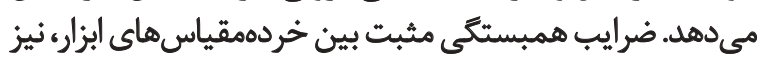
در جدول شماره 4 كزارش شده است مثبت بين
• ب/ • براى هر گويه، سادهترين و متناسبترين ساختار با الكوى

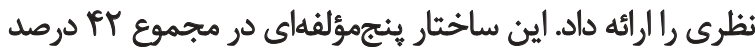

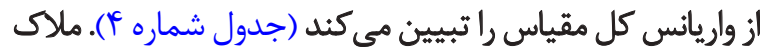

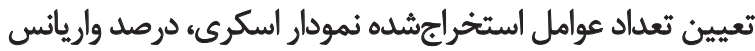
قابل تبيين توسط مؤلفهها و محتواي كويهها هر مؤلفه بوداري بررسى نتايج تحليل عاملى اكتشافى نشان داد مؤلفههاي

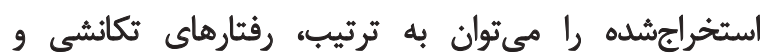

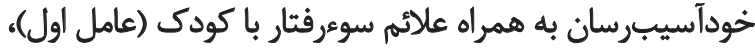

جدول و. ضرايب همبستكى خردهمقياسهاى نسخه الكترونيكى مقياس كزارش والدين از سلامت روانشئاختى دانشآموزان دوره متوسطه اول

\begin{tabular}{|c|c|c|c|c|c|}
\hline$\bullet$ & r & r & 1 & مؤلفهها & \\
\hline & & & 1 & رفتارهاى خودآسيبرسان و تكانشى به همراه علائم سوءرفتار با كودى & 1 \\
\hline & & 1 & .49000 & نقص در انجام تكاليف تحصيلى & r \\
\hline & 1 & $\cdot M N^{* *}$ & $+/ 999$ & بيش فعالى و نقص توجه & r \\
\hline 1 & $\cdot 1 \Delta \Lambda^{* *}$ &.$/ \Delta V^{*}$ &.$/ \propto q q^{\infty}$ & اضطراب & i \\
\hline $.19 Q^{* 0}$ & $. M=$ & $. M r=*$ &.$M I^{\infty}$ & |فسردمى | & $\Delta$ \\
\hline
\end{tabular}




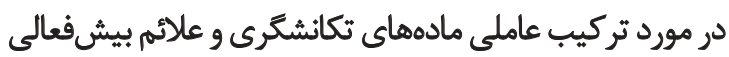

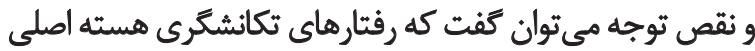

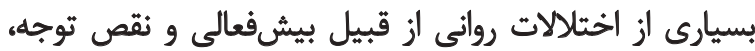

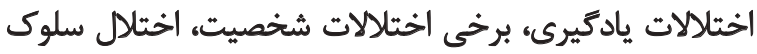

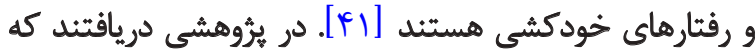

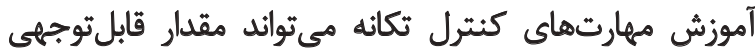

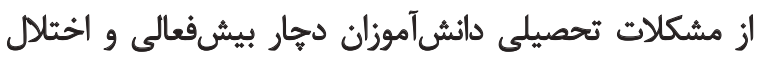

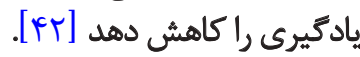

آموزش كنترل تكانه به طور معنادارى در افزايش توجه

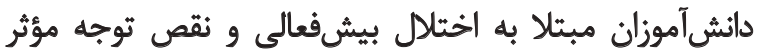

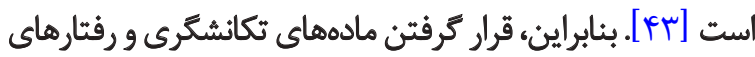

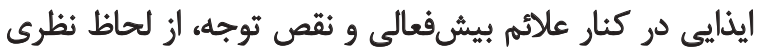

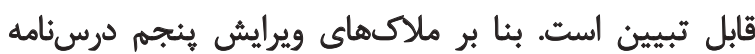

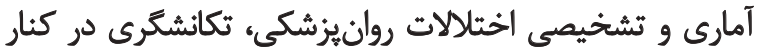

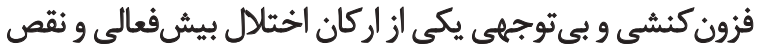

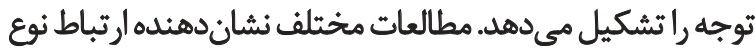

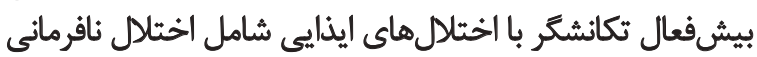

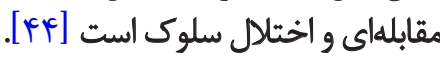

در نسخه كزارش والدين، رفتارهاى تكانشكَىى با رفتارهاى

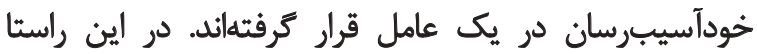

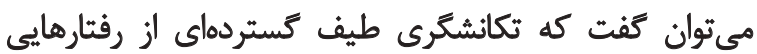

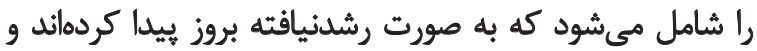

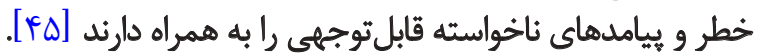

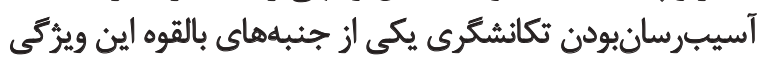

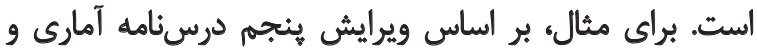

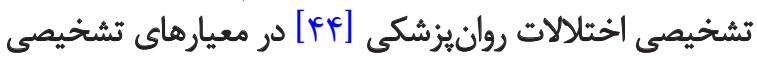

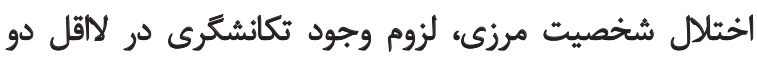

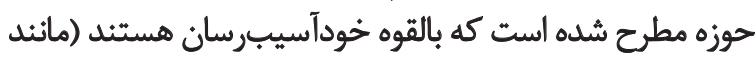

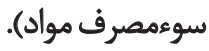

همجنين برخى ديكًر از معيارهاى تشخيصى اين اختلال ماندي

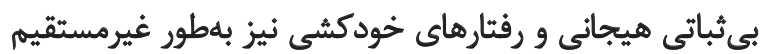

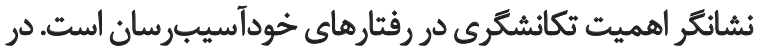

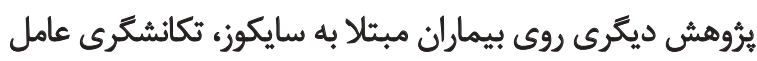

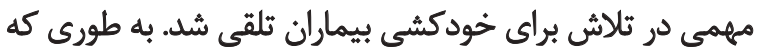

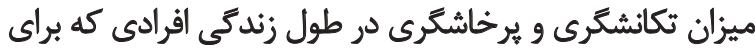

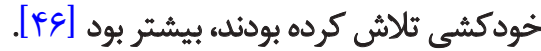

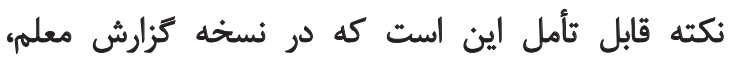

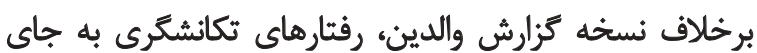

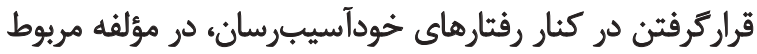

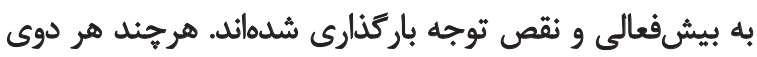

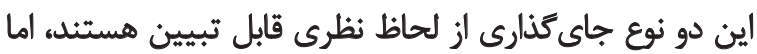

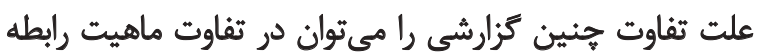

هدف از مطالعه حاضر، ساخت نسخه الكترونيكى مقياس

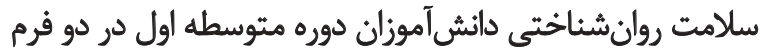

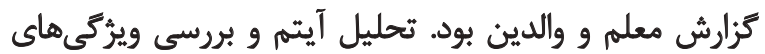

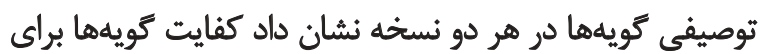

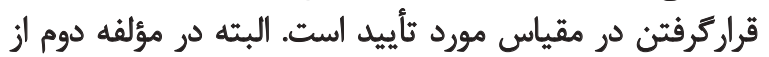

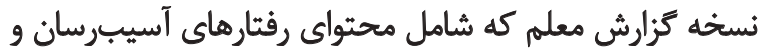

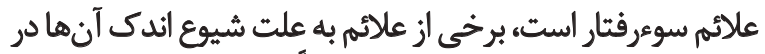

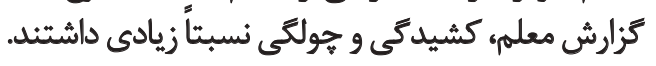

نتايج تحليل عاملى اكتشافى فرم كزارش معلم هم نشان

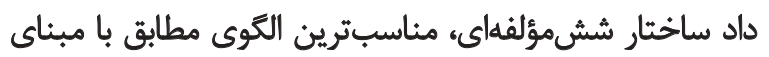

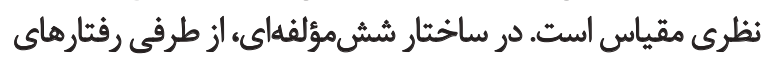

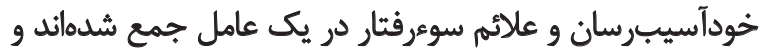

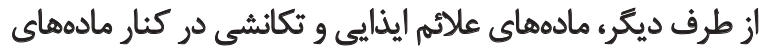

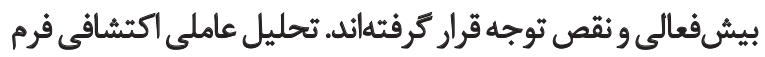

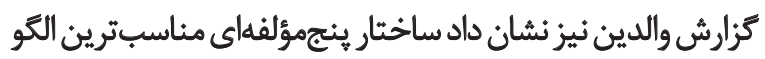

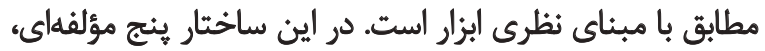

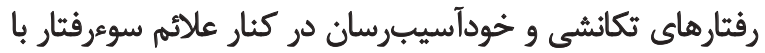
كودى در يك عامل جمع شدانداند.

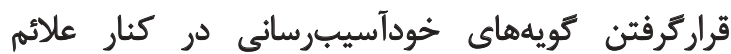

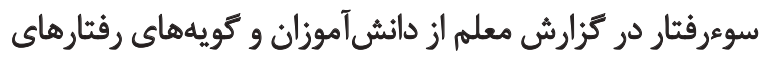

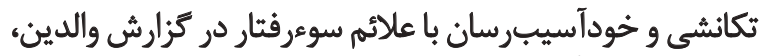

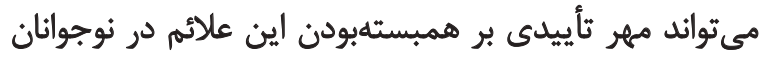

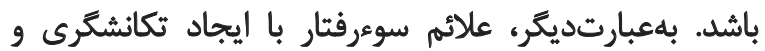

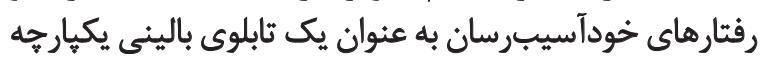

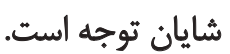

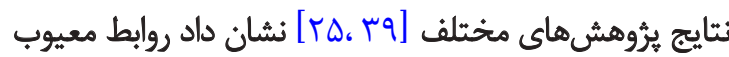

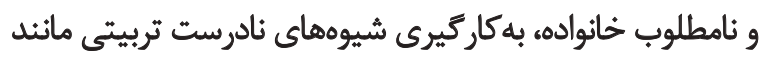

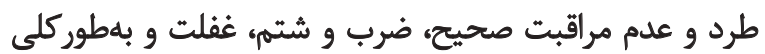

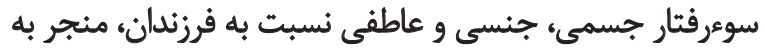

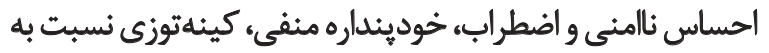

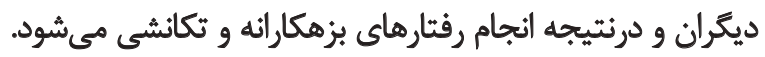

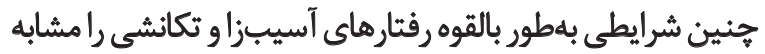

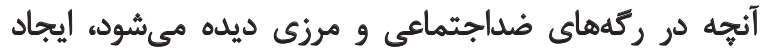

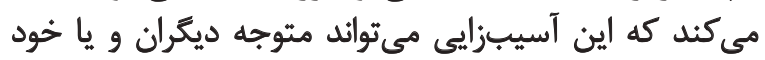
شخص شود [r. [r.

برايناساس، شرايط نامناسب در محيط كودكان و نوجوانان

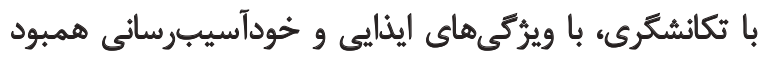

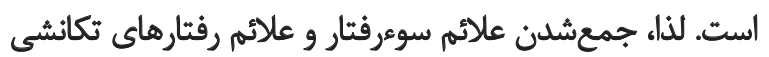

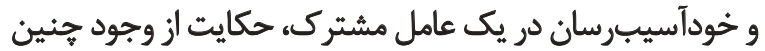
تابلوى بالينىاى در دانش آموزان بثوهش دارد 
مقياسهاى الكترونيكى سلامت روانشناختي دانشآموزان

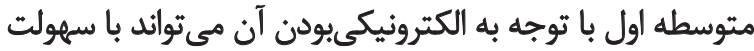

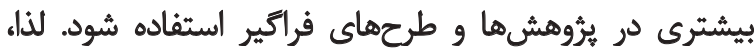

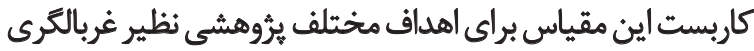

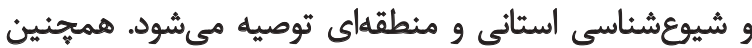

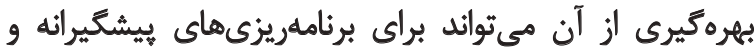

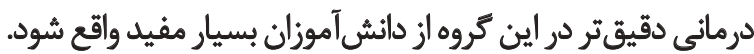

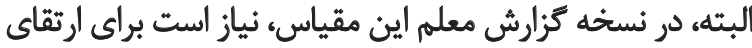

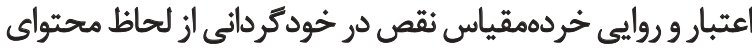
كويةها تجديدنظر شود.

از محدوديتهاى يُروهش حاضر مى توان به استفاده نكردن

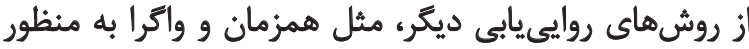

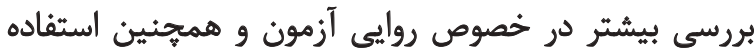

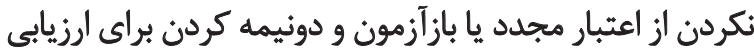

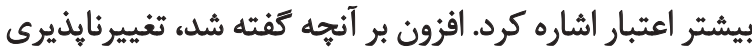

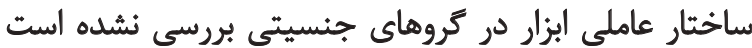

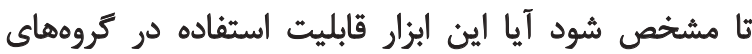
جنسيتى دختران و يسران را دارد يا خير.

همجنين، بيشنهاد مىشود از تحليلهاي مدرن بر اساس

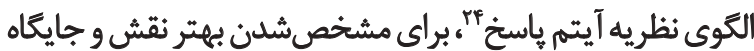

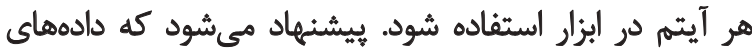

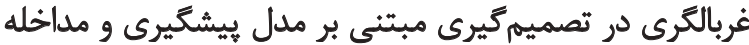

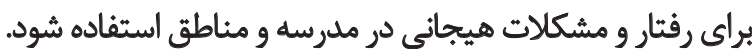

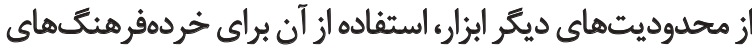

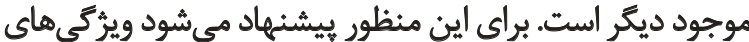
روانسنجى ابزار در اين مجموعهما بر درسى شودي.

\section{مالاحظات اخلاقى}

\section{ييروى از اصول اخلاق يثروهش}

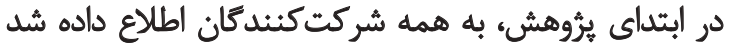

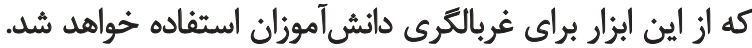

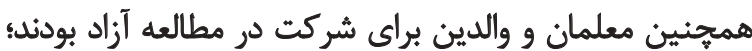

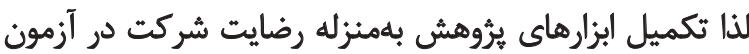

$$
\text { منظور شد. }
$$

مقاله حاضر از طرحى با عنوان الهطالعه مقدماتى ساخت

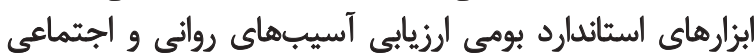

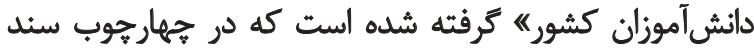
ائتلاف نظام مراقبتهاي اجتماعى ازئ دانشآ آموزان (نماد) ذيل

24. Item-Response Theory (IRT)

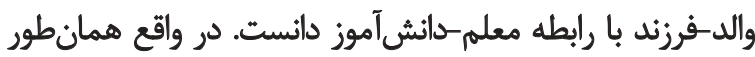

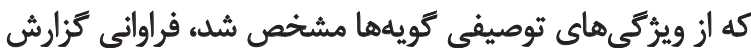

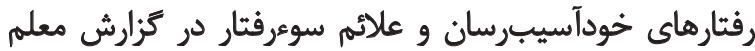

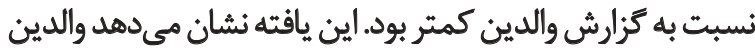

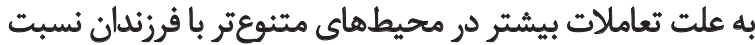

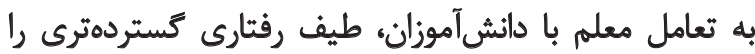

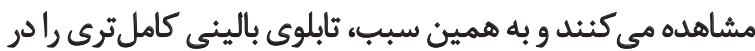

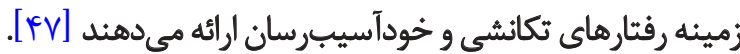
در ميان خردهمقياسها، خردهمقياس نقص در خودتردانى

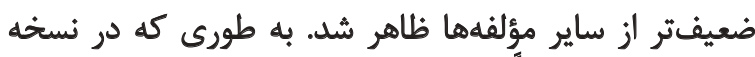

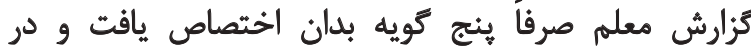

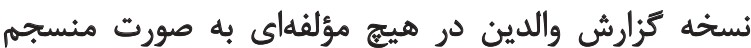

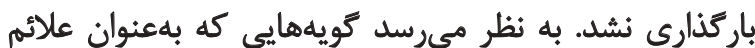

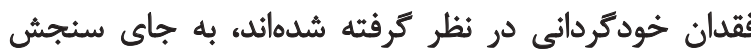
يك مفهوم همكون، مجموعهائ از كاركرئي

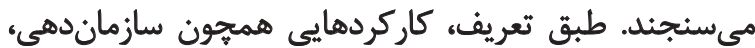

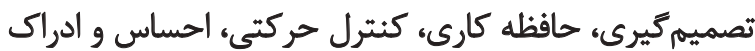

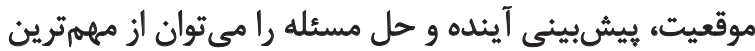

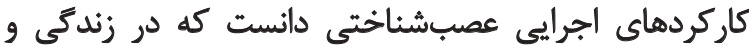

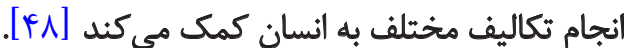

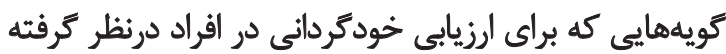

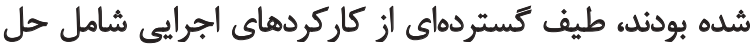

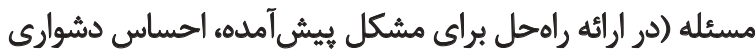

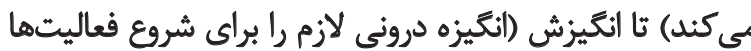

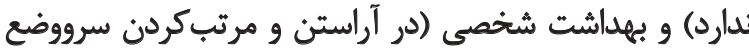

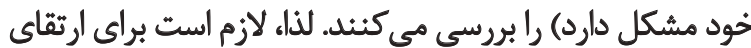

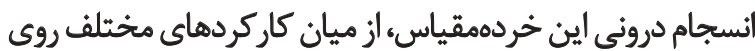

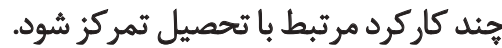

روابط بين خردممقياسهاى هر دو نسخه كزارش معلم و

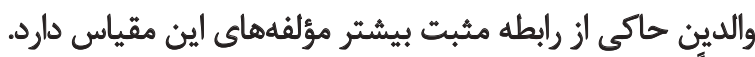

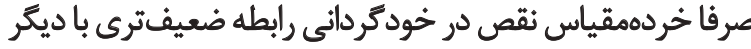

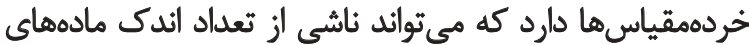

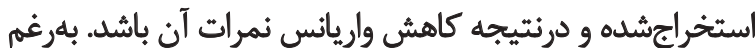

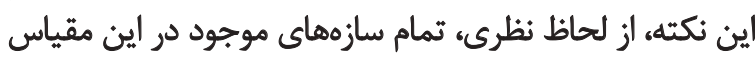

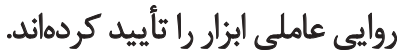

\section{نتيجليكيرى}

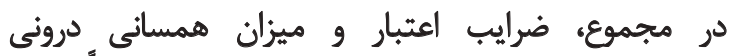

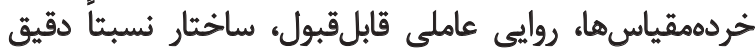

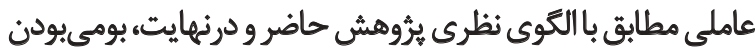

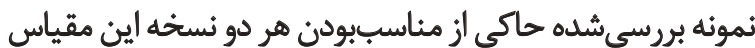

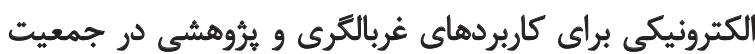

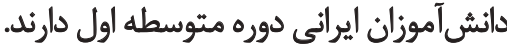


سند تقسيم كار ملى كنترل و كاهش آسيبهاي اجتماعى اجرا

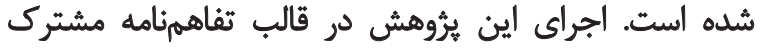

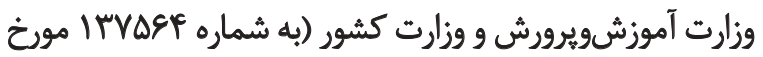

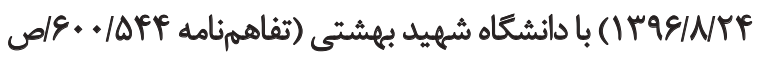

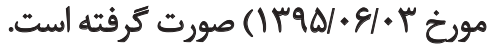

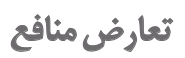

بين نويسندكان مقاله حاضر هيجكونه تعارض منافع مادى يا

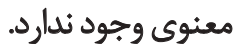

$$
\text { سياسكّزاوى }
$$

از تمامى معلمان و والدين كه ابزارهاى يُؤوهش را تكميل

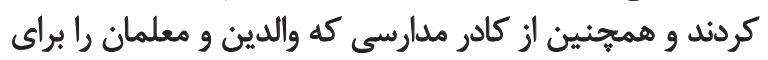

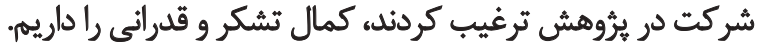




\section{References}

[1] Berk L. Developmental psychology [Y. Seyedmohammadi, Persian trans]. Tehran: Arasbaran; 2002.

[2] Aghamohammadian H, Mahjoub M, Hatefi M, Yazdi A. [The effectiveness of cognitive therapy in reducing obsessive-compulsive behaviors and anxiety in adolescents (Persian)]. Paper Presented at: The $6^{\text {th }}$ International Congress on Child and Adolescent Psychiatry. 17-19 September 2013, Tabriz, Iran.

[3] Wigfield A, Eccles, JS, Mac Iver D, Reuman DA, Midgley C. Transitions during early adolescence: Changes in children's domain-specific self-perceptions and general self-esteem across the transition to junior high school. Developmental Psychology. 1991; 27(4):552-65. [DOI:10.1037/0012-1649.27.4.552]

[4] Asnaashari N, Sheikholeslami, R. [The mediating role of emotional maturity in relationship between self-esteem and social anxiety among females adolescence (Persian)]. Journal of Psychological Models and Methods. 2016; 6(22):35-51.

[5] Hassanvand Amouzadeh M, Aghili MM, Hassanvand Amouzadeh $\mathrm{M}$. [The effect of cognitive-behavioral self-esteem group training on social anxiety and mental health in social anxious adolescent (Persian)]. Knowledge \& Research in Applied Psychology. 2013; 14(2):26-37.

[6] Rezaeian H, Mohsseni N, Mohammadi MR, Ghobari B, Sarmadi Z, Lavasani MG, et al. [Evaluation of casual model of family variables and self- understanding with conduct disorders (Persian)]. Hakim Research Journal. 2006; 9(3):32-7.

[7] Gesell, A. Infancy and human growth. NewYork: American Psycological Association; 1928. [DOI:10.1037/14664-000]

[8] Stiffler MC, Dever BV. Mental health screening at school: Instrumentation, implementation, and critical issues. Berlin: Spring; 2015.

[9] Polanczyk GV, Salum GA, Sugaya LS, Caye A, Rohde LA. Annual research review: A meta-analysis of the worldwide prevalence of mental disorders in children and adolescents. Journal of Child Psychology and Psychiatry. 2015; 56(3):345-65. [DOI:10.1111/ jcpp.12381] [PMID]

[10] Najafi M, Fuladchang M, Alizadeh H, Mohammadifar MA. [Prevalence of Attention Deficit Hyperactivity Disorder, Conduct Disorder and Oppositional Defiant Disorder (Persian)]. Research on Exceptional Children, 2009; 33(3):239-54

[11] Mashhadi A, Hamzeloo M, Bafandeh H. [The role of ADHD symptoms, sensation seeking and emotional-behavioral problems in predicating substance abuse in juvenile delinquents (Persian)]. Applied Psychological Research Quarterly. 2011; 2(4):45-60.

[12] Sayal K, Prasad V, Daley D, Ford T, Coghill D. ADHD in children and young people: prevalence, care pathways, and service provision. Lancet Psychiatry. 2018; 5(2):175-86. [DOI: 10.1016/ S2215-0366(17)30167-0

[13] Vilhena-Churchill N, Goldstein AL. Child maltreatment and marijuana problems in young adults: Examining the role of motives and emotion dysregulation. Child Abuse \& Neglect. 2014; 38(5):962-72. [DOI:10.1016/j.chiabu.2013.10.009] [PMID]

[14] ErfaniFar F, Zarrani F, Shokri O. [Emotional maltreatment and internalizing disorders in adolescent girls: The mediating role of emotion regulation (Persian)]. Journals Woman and Family Study; Forthcoming 2018. [DOI: 10.22051/JWFS.2017.13819.1352]
[15] Mikaeili N, Zamanloo Kh. [A study of the prevalence of child abuse and its prediction from parents' depression and anxiety, attachment styles and mental health of their adolescent boys (Persian)]. Research on Exceptional Children. 2012; 2(5):145-66.

[16] World Health Organization. Participant manual: IMAI oneday orientation on adolescents living with HIV. Geneva: World Health Organization; 2010.

[17] Veras JL, Ximenes RCC, de Vasconcelos FMN, Sougey EB. Prevalence of suicide risk among adolescents with depressive symptoms. Archives of Psychiatric Nursing. 2016; 30(1):2-6 [DOI:10.1016/j.apnu.2015.11.003] [PMID]

[18] Andriessen K, Rahman B, Draper B, Dudley M, Mitchell PB Prevalence of exposure to suicide: A meta-analysis of populationbased studies. Journal of Psychiatric Research. 2017; 88:113-120. [DOI:10.1016/j.jpsychires.2017.01.017] [PMID]

[19] Klassen RM, Krawchuk LL, Lynch SL, Rajani S. Procrastination and motivation of undergraduates with learning disabilities: A Mixed-Methods inquiry. Learning Disabilities Research \& Practice. 2008; 23(3):137-47. [DOI:10.1111/j.1540-5826.2008.00271.x]

[20] Narimani M, Mohammad Amini M, Zahedi A, Abdolghasemi A. A comparison of effectineness of training self-regulated learning strategies and problem-solving on academic motivation in male students with academic procrastination. Journal of School Psychology. 2015; 4(1):139-155.

[21] Javidi H, Asadi E, Ghasemi N. [The relationship between adolescent- parent conflict, resiliency with adolescent psychological problems Second and third level education in four areas of the city Shiraz (Persian)]. Psycological Studies. 2015; 11(1):75-96.

[22] Besharat M.[Resilience, vulnerability and mental health (Persian)]. Psychology Science. 2011; 6(24):373-83.

[23] Samani S, Jokar B. [A study on the reliability and validity of the short form of the depression anxiety stress scale (Dass-21) (Persian)]. Journal of Social Sciences and Humanities of Shiraz University. 2011; 26(3):65-76.

[24] Wilson JMG, Jungner G. Principles and practice of screening for disease. Switzerland: World Health Organization; 1968.

[25] Swanston HY, Parkinson PN, Oates RK, O'Toole BI, Plunkett AM, Shrimpton S. Further abuse of sexually abused children. Child Abuse \& Neglect. 2002; 26(2):115-27. [DOI:10.1016/S0145-2134(01)00311-8]

[26] Hill LG, Coie JD, Lochman JE, Greenberg MT. Effectiveness of early screening for externalizing problems: Issues of screening accuracy and utility. Journal of Consulting and Clinical Psychology. 2004; 72(5):809-20. [DOI:10.1037/0022006X.72.5.809] [PMID]

[27] Moyer VA, Calonge N, Teutsch SM, Botkin JR. Expanding newborn screening: process, policy, and priorities. Hastings Center Report. 2008; 38(3):32-9. [DOI:10.1353/hcr.0.0011] [PMID]

[28] Achenbach TM. Manual for the child behavior checklist 14-18 and 1991 profile. Burlington: General Hospital Psychiatry; 1991.

[29] Achenbach TM, Rescorla L. Manual for the ASEBA school-age forms \& profiles. Burlington: Research Center for Children, Youth and Families; 2001

[30] Piaget J, Inhelder B, Piaget, J. The growth of logical thinking from childhood to adolescence: An essay on the construction of formal operational structures. New York: Psychology Press; 1958. 
[31] Dadsetan P. [Developmental psychopatology (Persian)]. Tehran: SAMT; 2017.

[32] Jellinek MS, Murphy JM, Robinson J, Feins A, Lamb S, Fenton, T. Pediatric Symptom Checklist: screening school-age children for psychosocial dysfunction. The Journal of Pediatrics. 1988; 112(2):201-9. [PMID]

[33] Lane K, Kalberg JR, Bruhn AL, Driscoll SA, Wehby JH, Elliott, SN. Assessing social validity of school-wide positive behavior support plans: Evidence for the reliability and structure of the primary intervention rating scale. School Psychology Review. 2009; 38(1):135-44.

[34] Beck J, Beck AT, Jolly JB. Beck Youth Inventories of Emotional and Social Impairment: Depression Inventory for Youth, Anxiety Inventory for Youth, Anger Inventory for Youth, Disruptive Behavior Inventory for Youth, Self-concept Inventory for Youth: Manual. Journal of School Psychology. 2001; 42(4):333-40.

[35] Akbari Zardkhaneh S, Delavar A, Yaghubi H, Sohrabi Asmaroud F, Peyravi H. [Preliminary version of life skills scale for college students: item analysis, exploratory factorstructure and reliability coefficients (Persian)]. Applied Psychological Research Quarterly. 2012; 4(4):128-52.

[36] Jamali H, Sarraf Zadeh M, Asadi S. [Use of electronic questionnaires in surveys (Persian)]. Iranian Journal of Information Processing \& Management. 2015; 5(1):1384.

[37] McCoy S, Marks P. Using electronic surveys to collect data: Experiences from the field. Paper presented at: The Americas Conference on Information Systems. 2-5 August 2001; Boston, USA.

[38] Bryman A. Social research methods. Oxford: Oxford University Press; 2015.

[39] Pearson RH. Recommended sample size for conducting exploratory factor analysis on dichotomous data. Journal of Modern Applied Statistical Methods. 2008; 9(2):359-68. [DOI:10.22237/ jmasm/1288584240]

[40] Akbari-Zardkhaneh S, Sajadi S, Mansurkiaie N, Zanganeh A, Jallalat-Danesh M, Mahdavi M. [Second edition of parent form of psychological pathology for high school students (Persian)]. Applied Psychology; Forthcoming 2018.

[41] Akbari-Zardkhaneh S, Alavinejad S, Zanganeh A, Mansurkiaie N, Jallalat-Danesh M, Mahdavi M. [Parent form of psychological pathology for children: preliminary study of development and psychomentric properties (Persian)]. Journal of Child Mental Health. 2018; 4(4):152-64.

[42] Lemmey D, McFarlane J, Willson P, Malecha A. Intimate partner violence: Mothers' perspectives of effects on their children. The American Journal of Maternal/Child Nursing. 2001; 26(2):98103. [DOI:10.1097/00005721-200103000-00009] [PMID]

[43] Tahmasiyan K, Anari A, Fathabadi M. [Evaluation of Effective Maternal Characteristics in Behavioral Problems of 2-6 Years Old Children (Persian)]. Journal Management System. 1391; 7(29):89-104.

[44] Li CSR, Chen SH, Lin WH, Yang YY. Attentional blink in adolescents with varying levels of impulsivity. Journal of Psychiatr ic Research. 2005; 39(2):197-205. [DOI:10.1016/j.jpsyy chires.2004.06.003] [PMID]

[45] Preston AS, Heaton SC, McCann SJ, Watson WD, Selke G. The role of multidimensional attentional abilities in academic skills of children with ADHD. Journal of Learning Disabilities. 2009; 42(3):240-9. [DOI:10.1177/0022219408331042] [PMID]

[46] Mirnasab M M, Bonab BG. Effects of self-monitoring technique on inattentive behaviors of students with attention deficit hyperactivity disorder. Iranian Journal of Psychiatry. 2011; 6(2):84-86. [PMCID]

[47] American Psychiatric Association. Diagnostic and statistical manual of mental disorders. $5^{\text {th }}$ edition. Virginia: The American Psychiatric Association; 2013. [DOI:10.1176/appi. books.9780890425596]

[48] Waxman SE. A systematic review of impulsivity in eating disorders. European Eating Disorders Review. 2009; 17(6):408-25. [DOI:10.1002/erv.952] [PMID] 